\title{
Partial regulation in vertically differentiated industries*
}

\author{
Angela S. Bergantino ${ }^{\dagger} \quad$ Etienne Billette de Villemeur ${ }^{\ddagger} \quad$ Annalisa Vinella $^{\S}$
}

December 2009

\begin{abstract}
This paper offers theoretical foundations to price-and-quality cap regulation of recently liberalized utilities in which vertically differentiated services are provided by a regulated incumbent and an unregulated entrant competing in price and quality. The model may equally well represent competition across asymmetrically regulated industries. We establish that, in a variety of strategic settings, optimal weights in the cap targeted to the incumbent depend also on the market served by the entrant, despite the latter is not directly concerned by regulation. This calls for the possibility that regulators use information about the whole industries, rather than on the sole incumbents. We also evidence that the proposed regulatory scheme is robust to small errors in the estimation of the social value of quality.
\end{abstract}

Keywords: Price-and-quality cap; Partial regulation; Vertical differentiation J.E.L. Classification numbers: D43, L13, L51

\footnotetext{
${ }^{*}$ The paper was completed while the second author was visiting the University of Montréal, whose hospitality is gratefully acknowledged. We are indebted with Carlo Fiorio, David Martimort, Jérôme Pouyet, Bertrand Villeneuve, Ingo Vogelsang, an Associate Editor and two anonymous referees for helpful suggestions. We further acknowledge useful comments from participants at the XVIII SIEP Meeting (Pavia), the 5th Infraday (Berlin), the 4th Conference on Railroad Industry Structure, Competition and Investment (Madrid), the 47th SIE Meeting (Verona) and the 22nd EEA Meeting (Budapest) as well as from seminar participants at the University of Florence. All remaining errors are our own.

${ }^{\dagger}$ University of Bari, DSEMM (Italy)

${ }^{\ddagger}$ Toulouse School of Economics, IDEI and GREMAQ (France). Corresponding author. E-mail: Etienne.Devillemeur@TSE-fr.eu

${ }^{\S}$ University of Bari, DSEMM (Italy)
} 


\section{Introduction}

In markets where not only price (a monetary dimension) but also quality (a nonmonetary dimension) matters, pure price regulation does not yield overall desirable outcomes, in general (see, for instance, Armstrong and Sappington [3] and Sappington [30]).

Specifically, when firms are compelled to obey a price cap, they are induced to cut costs, which may translate into quality under-provision. This issue regards potentially all network industries in which a price cap is adopted. With reference to telecommunications, Vogelsang [32] observes that concerns about quality deterioration are widespread and that, indeed, such services are subject to price-cap regulation in most OECD countries and in several others. In fact, Rovizzi and Thompson [29] report that noticeable quality reduction was registered in British Telecom's services as soon as the company was submitted to a price cap, after privatization. According to Crew and Parker [17], among the various quality aspects that might suffer from price ceiling, most penalized seems to be service reliability, which is a crucial part of service value to end users ${ }^{1}$.

De Fraja and Iozzi [19] look for a way to use price cap in environments with relevant quality aspects, under the motivation that:

"Price-cap regulation (...) strikes a very good compromise between the theoretically rigorous foundation of the theory of optimal regulation (...) and the practitioner's requirement of the simple, easy-to-understand, easy-to-apply rule."

They integrate quality dimensions into the "standard" price cap, restructuring the latter as a price-and-quality cap. Characterizing the ideal composition of this incentive scheme with regard to multi-product monopolies, they find two essential results. The first result is that, in the index that enters the formula, the appropriate weights of the different prices are (proportional to) the optimal quantities of the products sold by the regulated firm. In other words, this cap does not differ from the "standard" price cap, where quality is not an issue ${ }^{2}$ (Brennan [12]; Laffont and Tirole [24]). The second result in De Fraja and Iozzi [19] is that quality weights in the "extended" cap should be equal to consumer marginal surplus evaluated at the optimal prices and qualities. Billette de Villemeur [8] obtains similar findings with reference to monopolistic airline industries, where relevant dimensions are price and service frequency ${ }^{3}$.

\footnotetext{
${ }^{1}$ Service reliability introduces an element of heterogeneity even in electricity, a product that is otherwise perfectly homogeneous. Specifically, in power sectors, reaction lags and supply interruptions are relevant quality dimensions (compare Crampes and Moreaux [14]).

${ }^{2}$ Standard price cap may find specifications according to the context. For instance, Billette de Villemeur, Cremer, Roy and Toledano [9] characterize a price-cap scheme that fits postal sector features.

${ }^{3}$ Under monopoly (though not in other frameworks), service frequency is equivalent to a pure quality attribute.
} 
These findings do not need to extend to vertically differentiated oligopolies in which regulation concerns one sole firm. With regard to non-monopolistic sectors, it is known that, if a regulated incumbent competes with an unregulated passive fringe, then total market quantities are the optimal weights in the pure price cap only if fringe profits are not included in social welfare. By contrast, if fringe profits are taken to contribute to social welfare, then appropriate weights relate to the optimal quantities of the sole regulated firm. These results are found by Brennan [12], who further acknowledges that, when competitors are not price-takers, a different recipe is required. Yet, the author does not formally provide such a recipe, even just for pure price cap.

In the present article, we provide theoretical foundations to price-and-quality cap regulation ${ }^{4}$ of oligopolies where a regulated incumbent competes in price and quality with one (or few) strategic rival(s), which operate(s) unregulated. By doing so, we extend to oligopolistic settings the literature about price-and-quality regulation through cap schemes, which has focused on monopolistic markets so far.

The framework we consider, namely an oligopoly where the sole incumbent is regulated, closely reflects the most common evolution that formerly public utilities have recently undergone as a result of the liberalization process. Typically, in those sectors, the former monopolist goes subject to regulatory control, whereas the (few) competitors that have entered after liberalization operate unregulated, despite exerting market power in the seek for profits. Our model is meant to stylize concentrated and partially regulated industries of this sort. To fix ideas, one may think about competition between regulated telephone companies and unregulated cable voice-over-Internet-Protocol or wireless cellular companies in voice telephony. One may further consider competition between regulated and unregulated cable pay-televisions. Actually, the model may equally well represent competition across asymmetrically regulated industries. One instance is inter-modal competition between regulated train operators and deregulated air carriers in European transport industries.

To capture the relevance of quality provision and motivate quality regulation, we represent a market where vertically differentiated services are supplied to consumers exhibiting heterogeneous quality valuations. Our choice to model vertical (rather than horizontal) differentiation follows from the observation that, in the industrial contexts we refer to, consumers tend to share the same quality ranking, e.g. they perceive the product provided by the incumbent as superior to the product(s) offered by the competitor(s). For instance, in voice telephony, the services provided by telephone companies are generally more reliable than those provided by cable companies. Similarly, most of the times, air transport is considered to be more comfortable and reliable than rail transport. In turn, regulated cable televisions typically broadcast higher quality programmes and propose less advertising than unregulated competitors.

Importantly, the possibility that quality be regulated follows from the circumstance that it is observable and verifiable. This is actually the case in nearly all network industries.

\footnotetext{
${ }^{4}$ The effects of price ceilings on service quality has already been investigated both theoretically and empirically (see, for instance, Sappington [31] and Weisman [34]).
} 
Insisting on the aforementioned sectors, it is indeed possible to observe and collect data about connection interruptions in voice telephony, advertising frequency and programme content in cable TV services, travel time and departure/arrival delays in transport services. This is why, as the literature has pointed out, in network industries, service quality is often heavily regulated, whereas infrastructure quality, which is hardly observable and verifiable, remains unregulated in general (see Martimort and Sand-Zantman [27], for instance). As observed by a referee, data collection can be expensive. However, real-world practice seems to suggest that it is worth its $\operatorname{cost}^{5}$.

Furthermore, it is frequently the case that the quality of the goods and/or services the utilities provide (though not the inner quality of the network infrastructures) can be adjusted in the short run. To reflect this circumstance, in our model we take quality to be as flexible as price. Yet, we also discuss the consequences of quality being a longer-run decision variable ${ }^{6}$.

We begin by characterizing the optimal price-and-quality pair to be decentralized by the regulator. In the presence of strategic rivals that remain unregulated, the relevant benchmark is given by the equilibrium price-and-quality pair of a market game in which a welfare-maximizing firm competes with one (or more) profit-maximizing rival(s), under the requirement that its profit be non-negative. From this perspective, our work is reminiscent of the mixed oligopoly models in which a public firm competes with one (or more) private operator(s) under budget balance ${ }^{7}$. We characterize the price-and-quality equilibrium for two kinds of market game, namely a Stackelberg game, in which the incumbent/leader chooses price and quality anticipating the reaction of the entrant/follower, and a NashCournot game, in which firms make choices simultaneously taking the rival's as given. In so doing, we account for the circumstance that the regulated firm may or may not enjoy a strategic advantage vis-à-vis the unregulated entrant, depending upon how competition, on one side, and regulation, on the other, affect its market position and commitment ability $^{8}$.

\footnotetext{
${ }^{5}$ To mention only a few examples, data about TV channels (types of broadcast, audience shares), advertising (spot duration, frequency) and programmes (duration, content) are largely available. For instance, in the UK, the Office of Communications (Ofcom) systematically circulates such data through surveys and reviews (see also Carat [13] for information about EU countries). As for transport services, in the U.S., the Bureau of Transportation Statistics of the Research and Innovative Technology Administration provides detailed information about departure and arrival delays for a variety of transportation modes (aviation, maritime, highway, transit, rail). Similarly, in France, the Observatoire des retards du transport aérien, as managed by the Direction générale de l'aviation civile in cooperation with airlines and airports, collects and publishes data on flight punctuality. In Italy, the regulated rail company is currently compelled to disclose information about delays at arrival. Furthermore, delays are being increasingly monitored by consumers' associations and other concerned institutions (see, for instance, the report by Legambiente [26], based on Censis data, about the situation of Italian railways commuter transport).

${ }^{6}$ We thank an Associate Editor for bringing this case to our attention.

${ }^{7}$ Within the domain of literature about mixed oligopolies, Bös [11] reaches the conclusion that a public firm facing the requirement to operate at zero profit should stick to a modified Ramsey-pricing rule. In turn, exploring a homogeneous-product Stackelberg game with the public firm in the leader's position, Beato and Mas Colell [5] show that the solution to this game involves average-cost pricing for the public firm. The latter anticipates the competitor's policy choices, setting quantity so as to break even at equilibrium.

${ }^{8}$ On one hand, regulation may reinforce the commitment ability of the firm by limiting its operational flexibility. On the other hand, repeated revisions may progressively inhibit that ability. Subtle is the relationship between the incumbent's strategic behaviour and the regulator's possibility to pursue different
} 
Once characterized the Stackelberg and the Nash-Cournot equilibrium that constitute the two possible regulatory targets, we demonstrate how each of them can be decentralized by means of a properly structured price-and-quality cap targeted to the sole incumbent. Importantly, it turns out that the optimal weights to be attached to the latter's price and quality have exactly the same composition, whatever the benchmark. These weights should be set taking into account not only the market served by the incumbent, but also the market(s) covered by the unregulated competitor(s). It further emerges that, when the Stackelberg target is pursued, the unregulated market(s) should also be considered to tighten/relax the global ceiling. These findings involve that, at the implementation stage, regulatory bodies of liberalized industries should not be restricted to access and use information about the sole regulated firms. They should rather be allowed to extract and make use of information about the overall industry. This provides a neat argument against the enforcement of norms that prevent regulators from basing their policies on information about unregulated markets and/or activities.

Still concerning implementation, we suggest that both the Stackelberg and the NashCournot equilibrium be progressively approached by applying the regulatory scheme iteratively over time, hinging on past data about market activities. We show that, when this strategy is indeed followed, in either of the relevant frameworks, the quality-adjusted price cap we propose exhibits the desirable property of being robust to small errors in the practical determination of the weights to be attached to the regulated firm's price and quality.

The remainder of the article is organized as follows. In section 2 we present the framework. In section 3 we characterize the regulatory benchmarks, i.e. the equilibrium price-and-quality pair of a Stackelberg and of a Nash-Cournot mixed duopoly. In section 4 we show how either target can be decentralized by means of an appropriate priceand-quality cap. In section 6 we discuss the generality of our results and provide a few concluding remarks. Most of the mathematical details are relegated to an Appendix.

\section{The model}

We consider an industry where two firms, namely an incumbent and an entrant, provide vertically differentiated products. The incumbent, denoted $I$, is subject to regulation. The competitor, denoted $E$, is not.

Firms' strategic variables are price and quality (respectively, $p_{k}$ and $q_{k}, k=I, E$ ). We suppose that both operators choose their own price and quality simultaneously ${ }^{9}$. We further take price and quality to be observable and verifiable.

We explore two kinds of market game, namely the Stackelberg and the Nash-Cournot game. In the former, firm $I$, the leader, anticipates the impact of its own price-and-quality choice on the decision that firm $E$, the follower, will make. This framework closely repre-

(more or less ambitious) targets. This opens delicate issues that would deserve specific attention but are beyond the scope of the present work.

${ }^{9}$ See Section 5 for a discussion of the case in which firms choose quality before price. 
sents situations in which the incumbent of a previously fully regulated industry does enjoy a strategic advantage vis-à-vis the newcomer. In the Nash-Cournot game, each provider chooses its own price and quality taking the competitor's as given. This framework mirrors contexts in which, unlike in the previous ones, the incumbent lacks commitment ability vis-à-vis the unregulated competitor.

The goods that firms provide are perfect substitutes, except for the difference in quality. Consumers are heterogeneous in their valuation for quality, which is represented by a parameter $\theta$. More precisely, sticking to a quasi-linear framework, we assume that the net surplus a consumer of characteristic $\theta$ derives from the consumption of $x$ units of quality $q$ bought at unit price $p$ is written

$$
v_{\theta}(x, p, q)=u(x)-(p-\theta q) x
$$

with $u(x)$ increasing and concave in the argument. The parameter $\theta$ is distributed over the interval $[\underline{\theta}, \bar{\theta}]$, with $\bar{\theta}>\underline{\theta} \geq 0$ and according to a continuous density function $f(\theta)$. The associated cumulative distribution function is denoted $F(\theta)$. Given her quality valuation, a $\theta$-consumer patronizing firm $k \in\{I, E\}$ faces the so-called generalized price $\widetilde{p}_{k}(\theta) \equiv$ $\left(p_{k}-\theta q_{k}\right)$, that is the unit price $p_{k}$ net of the benefits $\theta q_{k}$ associated with product quality. A $\theta$-consumer prefers to purchase the good from firm $k$, rather than from firm $j \neq k \in$ $\{I, E\}$, whenever by doing so he/she bears a smaller generalized price $\left(\widetilde{p}_{k}(\theta)<\widetilde{p}_{j}(\theta)\right)$. Observe that, by construction, no consumer finds it beneficial to patronize both firms, as usual in environments with vertical differentiation.

We suppose, without loss of generality, that firm $k$ sells a product of higher quality at higher price $\left(q_{k}>q_{j}, p_{k}>p_{j}\right)$. The marginal consumer, who is indifferent between the two operators, is characterized by the parameter value

$$
\theta_{m} \equiv \frac{p_{k}-p_{j}}{q_{k}-q_{j}}
$$

Thus, individuals whose $\theta$ exceeds $\theta_{m}$ patronize firm $k$, whereas individuals whose $\theta$ is smaller than $\theta_{m}$ patronize firm $j$. We abstract from the possibility that some of the potential consumers abstain from making any purchase, an unlikely case for some "basic" services like telecommunications or daily transport services.

\subsection{Consumer valuation of quality, demand and surplus}

The demand of a $\theta$-consumer is pinned down by maximizing (1) with respect to $x$. This yields

$$
\frac{\partial u}{\partial x}=\widetilde{p}_{k}(\theta)
$$

\footnotetext{
${ }^{9}$ In a more general formulation, one could allow the marginal valuation of quality to depend on the quality level, namely $\theta(q)$. However, imposing the restriction that $\theta(q)=\theta, \forall q$, does not affect the very nature of results, as long as variations in the quality level do not yield significant variations in the marginal valuation of quality.
} 
where $\widetilde{p}_{k}(\theta)=\arg \min \left\{\widetilde{p}_{I}(\theta), \widetilde{p}_{E}(\theta)\right\}$. Individual consumption $x_{k}\left(p_{k}, q_{k} ; \theta\right)$ appears to be a function of the sole generalized price, $\widetilde{p}_{k}(\theta)$, of the consumed commodity. As $\widetilde{p}_{k}(\theta)$ decreases with $\theta$ and provided $\widetilde{p}_{k}\left(\theta_{m}\right)=\widetilde{p}_{j}\left(\theta_{m}\right)$, the ranking of consumers in terms of quality valuation is reflected in their ranking in terms of individual consumption $x_{\theta}$. Formally, $x_{\theta_{1}} \leq x_{\theta_{2}}$ whenever $\theta_{1} \leq \theta_{2}$.

Relying upon (3), it is possible to establish the relationship between the impacts on consumption of marginal changes in price and quality. To see this, observe first that (3) holds for any $p_{k}$ and $q_{k}$. Differentiating both sides with respect to $p_{k}$ and to $q_{k}$ and combining the two equations, we obtain

$$
\frac{\partial x_{k} / \partial q_{k}}{-\partial x_{k} / \partial p_{k}}=\theta, \quad \forall k \in\{I, E\}
$$

This evidences that, for the demand of a $\theta$-consumer to remain unchanged as price $p_{k}$ is increased by one unit, quality $q_{k}$ should be raised by an amount equal to the individual marginal valuation for quality, namely $\theta$. It also follows that a consumer with a strictly higher quality valuation patronizing the same firm would consider an increase in $\left(p_{k}, q_{k}\right)$ that leaves a $\theta$-consumer indifferent as strictly beneficial. Conversely, a consumer with a strictly lower valuation would find it detrimental. Opposite appreciations would arise if a decrease in $\left(p_{k}, q_{k}\right)$ that leaves a $\theta$-consumer indifferent were considered.

Firms' aggregate demands are immediately obtained by summing over the relevant ranges of $\theta$. Under the hypothesis that firm $k$ serves high-valuation consumers and firm $j$ low-valuation consumers, we formally have

$$
\begin{aligned}
& X_{j}(\mathbf{p}, \mathbf{q})=\int_{\underline{\theta}}^{\theta_{m}(\mathbf{p}, \mathbf{q})} x_{j}\left(p_{j}, q_{j} ; \theta\right) f(\theta) d \theta \\
& X_{k}(\mathbf{p}, \mathbf{q})=\int_{\theta_{m}(\mathbf{p}, \mathbf{q})}^{\bar{\theta}} x_{k}\left(p_{k}, q_{k} ; \theta\right) f(\theta) d \theta
\end{aligned}
$$

where $\mathbf{p}$ and $\mathbf{q}$ denote the vector of prices and qualities respectively. Demands display the rather standard properties that we briefly recall hereafter. For any $k, j \in\{I, E\}$ :

1. $\left(\partial X_{k} / \partial p_{k}\right)<0$ : demand for firm $k^{\prime}$ s product decreases with its own price $p_{k}$;

2. $\left(\partial X_{k} / \partial q_{k}\right)>0$ : demand for firm $k^{\prime}$ s product increases with its own quality $q_{k}$;

3. $\left(\partial X_{k} / \partial p_{j}\right)>0$ : demand for firm $k^{\prime}$ s product increases with the rival price $p_{j}$;

4. $\left(\partial X_{k} / \partial q_{j}\right)<0$ : demand for firm $k^{\prime}$ s product decreases with the rival quality $q_{j}$.

It is also straightforward to obtain aggregate consumer surplus as a function of prices and qualities. At this aim, we plug individual demands, as pinned down by (3), into the surplus function (1) and sum over the relevant ranges of $\theta$. This ultimately returns

$$
V(\mathbf{p}, \mathbf{q})=\int_{\underline{\theta}}^{\theta_{m}(\mathbf{p}, \mathbf{q})} v_{\theta}\left(x_{\theta}, p_{j}, q_{j}\right) f(\theta) d \theta+\int_{\theta_{m}(\mathbf{p}, \mathbf{q})}^{\bar{\theta}} v_{\theta}\left(x_{\theta}, p_{k}, q_{k}\right) f(\theta) d \theta .
$$




\subsection{Technologies and profits}

We denote $C_{k}\left(X_{k}, q_{k}\right)$ the cost function of firm $k \in\{I, E\}$. This function is assumed to be continuous and increasing in both production level and quality. In formal terms $\left(\partial C_{k} / \partial X_{k}\right)>0$ and $\left(\partial C_{k} / \partial q_{k}\right)>0, \forall k \in\{I, E\}$. We further assume that $\lim _{q_{k} \rightarrow+\infty} C_{k}\left(X_{k}, q_{k}\right)=+\infty$. This says that high quality products are so costly to improve that perfect products $\left(q_{k} \rightarrow+\infty\right)$ are never actually offered on the market. We finally assume that the firms never find it profitable to decrease the quality of their products down to zero. Taken together, these hypotheses ensure an interior solution to the determination of quality. Firm $k^{\prime}$ s profit function is written

$$
\pi_{k}(\mathbf{p}, \mathbf{q})=p_{k} X_{k}-C_{k}\left(X_{k}, q_{k}\right), \quad \forall k \in\{I, E\}
$$

\section{Characterization of the regulatory benchmark}

As a first step of the analysis, we need to characterize the (constrained) optimal priceand-quality bundle, which the regulator should take as a target. This is the bundle that would arise at the equilibrium of a mixed duopoly where a welfare-maximizing (public) firm were to compete with a profit-maximizing (private) firm, under a non-negative profit constraint. We characterize this bundle in the two contexts of our interest, namely the Stackelberg and the Nash-Cournot game.

\subsection{The profit-maximizing $\left(p_{E}, q_{E}\right)$-pair}

We begin by exploring the price-and-quality choice of the profit-maximizing competitor. In either game, firm $E$ takes the price and quality of firm $I$ as given and optimizes its own accordingly. Let $\varepsilon_{E} \equiv\left(p_{E} / X_{E}\right)\left(-\partial X_{E} / \partial p_{E}\right)$ be (the absolute value of) the demand elasticity with respect to its own price.

Lemma The price-and-quality bundle that maximizes $\pi_{E}(\mathbf{p}, \mathbf{q})$ is characterized by the following pair of conditions:

$$
\begin{aligned}
\frac{p_{E}-\left(\partial C_{E} / \partial X_{E}\right)}{p_{E}} & =\frac{1}{\varepsilon_{E}} \\
p_{E} \frac{\partial X_{E}}{\partial q_{E}} & =\frac{\partial C_{E}}{\partial q_{E}}+\frac{\partial C_{E}}{\partial X_{E}} \frac{\partial X_{E}}{\partial q_{E}} .
\end{aligned}
$$

Equation (8) is the standard Lerner formula. It evidences that firm $E$ acts as a monopolist vis-à-vis the "residual demand", $X_{E}$. Moreover, according to equation (9), quality $q_{E}$ is chosen so that marginal returns from quality improvements (the left-hand side) equate marginal costs (the right-hand side). The latter are expressed by the sum of the direct costs of quality (the first term) and its indirect costs (as reflected in the second term) that follow from the demand increments induced by quality raise. 
Rearranging (9) and combining it with (8) yields

$$
\frac{\partial X_{E} / \partial q_{E}}{-\partial X_{E} / \partial p_{E}}=\frac{1}{X_{E}} \frac{\partial C_{E}}{\partial q_{E}}
$$

Interestingly, by analogy with equation (4), the ratio in the left-hand side of (10) can be interpreted as the aggregate marginal valuation of quality by firm $E^{\prime}$ s clients. In turn, the right-hand side of (10) represents the average cost of a marginal increase in quality for this same firm. Although firm $E$ is a profit maximizer, no distortion is introduced by the choices it makes in terms of quality. Given consumer valuation, further quality improvement would not appear to be worth its $\operatorname{costs}^{10}$.

\subsection{The welfare-maximizing $\left(p_{I}, q_{I}\right)$-pair}

We now move to characterize the regulatory target. Recall that this is the price-andquality bundle that firm $I$, the regulated incumbent, should implement so as to pursue social interests without incurring budgetary losses. In formal terms, both in the Stackelberg and in the Nash-Cournot framework, the optimal $\left(p_{I}, q_{I}\right)$-pair is pinned down by maximizing the social welfare function

$$
W(\mathbf{p}, \mathbf{q})=V(\mathbf{p}, \mathbf{q})+\pi_{I}(\mathbf{p}, \mathbf{q})+\pi_{E}(\mathbf{p}, \mathbf{q})
$$

subject to the non-negative profit constraint

$$
\pi_{I}(\mathbf{p}, \mathbf{q}) \geq 0
$$

knowing that $p_{E}$ and $q_{E}$ obey the rules in $(8)$ and $(9)^{11}$. Let $\lambda$ the Lagrange multiplier associated with (12). Further denote

$$
\begin{aligned}
& \tilde{\theta}_{k} \equiv \int_{\theta_{m}(\mathbf{p}, \mathbf{q})}^{\bar{\theta}} \frac{x_{k}\left(p_{k}, q_{k} ; \theta\right)}{X_{k}} \theta f(\theta) d \theta, \\
& \tilde{\theta}_{j} \equiv \int_{\underline{\theta}}^{\theta_{m}(\mathbf{p}, \mathbf{q})} \frac{x_{j}\left(p_{j}, q_{j} ; \theta\right)}{X_{j}} \theta f(\theta) d \theta,
\end{aligned}
$$

the weighted average of quality valuations by the clients of firm $k$ and $j$ respectively. Taking into account that the incumbent foresees the impact of its decisions on the entrant's in the Stackelberg game, whereas it behaves myopically in the Nash-Cournot game, we can state the following proposition ${ }^{12}$.

\footnotetext{
${ }^{10}$ This does not mean that consumer and firm's objectives are perfectly aligned, even when attention is restricted to the quality dimension. In fact, were the price lower, the demand would be larger. As a result, the average cost of quality would be smaller, calling for a strict improvement in terms of quality.

${ }^{11}$ Because the entrant is not subject to regulatory control, the regulator does not need to be concerned with the financial viability of firm $E$. Yet, as we assume that the regulated firm does face an unregulated competitor, we implicitly take the entrant's profit to be non-negative at the incumbent's welfare-maximizing price and quality.

${ }^{12}$ See Appendix A.1.1 for mathematical details.
} 
Proposition 1 Under the Lemma, the incumbent's price-and-quality bundle that maximizes (11) subject to (12) is characterized by the following pairs of conditions:

a) in the Stackelberg game:

$$
\begin{aligned}
\frac{d \pi_{I}}{d p_{I}}= & \frac{1}{1+\lambda}\left[X_{I}-X_{E}\left(\frac{\partial X_{E} / \partial p_{I}}{-\partial X_{E} / \partial p_{E}}\right)\right. \\
& \left.+X_{E} \frac{d p_{E}}{d p_{I}}-\widetilde{\theta}_{E} X_{E} \frac{d q_{E}}{d p_{I}}\right] \\
\frac{d \pi_{I}}{d q_{I}}= & \frac{-1}{1+\lambda}\left[\widetilde{\theta}_{I} X_{I}+X_{E}\left(\frac{\partial X_{E} / \partial q_{I}}{-\partial X_{E} / \partial p_{E}}\right)\right. \\
& \left.-X_{E} \frac{d p_{E}}{d q_{I}}+\widetilde{\theta}_{E} X_{E} \frac{d q_{E}}{d q_{I}}\right]
\end{aligned}
$$

b) in the Nash-Cournot game:

$$
\begin{aligned}
& \frac{\partial \pi_{I}}{\partial p_{I}}=\frac{1}{1+\lambda}\left[X_{I}-X_{E}\left(\frac{\partial X_{E} / \partial p_{I}}{-\partial X_{E} / \partial p_{E}}\right)\right] \\
& \frac{\partial \pi_{I}}{\partial q_{I}}=\frac{-1}{1+\lambda}\left[\widetilde{\theta}_{I} X_{I}+X_{E}\left(\frac{\partial X_{E} / \partial q_{I}}{-\partial X_{E} / \partial p_{E}}\right)\right] .
\end{aligned}
$$

As the incumbent's objective is to attain the highest feasible welfare level, both equation (13) and (14) and equation (15) and (16) embody the variations that, respectively, a raise in price $p_{I}$ and in quality $q_{I}$ induces both in net consumer surplus $(V)$ and in the competitor's profit $\left(\pi_{E}\right)$.

Specifically, in (13) and (15), the term $X_{I}$ captures the direct marginal impact of a raise in $p_{I}$ on $V$, the term $X_{E}\left(\frac{\partial X_{E} / \partial p_{I}}{-\partial X_{E} / \partial p_{E}}\right)$ that on $\pi_{E}$. The latter is the product of the marginal rates at which the incumbent's and the competitor's price are to be substituted for the size of the market served by the competitor not to vary, and the very same market size, $X_{E}$. Similarly, in (14) and (16), the term $\widetilde{\theta}_{I} X_{I}$ captures the direct marginal impact of a raise in $q_{I}$ on $V$, the term $X_{E}\left(\frac{\partial X_{E} / \partial q_{I}}{-\partial X_{E} / \partial p_{E}}\right)$ that on $\pi_{E}$. The latter is the product of the marginal rate at which the incumbent's quality and the competitor's price are to be substituted for the size of the market served by the competitor not to vary, and, again, the very same market size, $X_{E}$. This evidences the relevance of cross-price and crossquality effects $\left(\partial X_{E} / \partial p_{I}\right.$ and $\left.\partial X_{E} / \partial q_{I}\right)$ for the determination of the incumbent's optimal price-and-quality pair.

Besides, in the Stackelberg game, strategic interactions across firms are accounted for. In (13) and (14), they are captured by the terms $\left(d p_{E} / d p_{I}\right),\left(d q_{E} / d p_{I}\right),\left(d p_{E} / d q_{I}\right)$ and $\left(d q_{E} / d q_{I}\right)$. The presence of these terms is due to the circumstance that variations in the price and quality of the market leader also affect consumer utility through the impact on its rival's price and quality. This involves both a volume and a quality appreciation effect. The former is expressed by the follower's demand, with which the terms under scrutiny are systematically weighed. The latter is measured by the average valuation of quality of 
the follower's clients, with which the terms are also weighed whenever interactions with quality $q_{E}$ are concerned. Of course, these terms do not appear in (15) and (16) because, under Nash-Cournot competition, the incumbent does not anticipate the effect of its own decisions on the competitor's.

The apparent complexity of the conditions in Proposition 1, especially as far as the Stackelberg setting is concerned, may induce one to consider the definition of the optimal price and quality as a purely theoretical exercise, with no practical value. If the optimal bundle does not find an explicit expression, then exact implementation is indeed likely to be beyond reach. This makes more striking the results we present hereafter.

\section{Decentralization through a quality-adjusted price cap}

In Proposition 1, we have identified the benchmark the regulator should effect in the marketplace. It is represented by (13) and (14) when firm $I$ acts as a Stackelberg leader. It is given by (15) and (16) when firm $I$ plays à la Nash-Cournot. Decentralization requires that the regulator adopt an appropriate policy device. For either setting, we hereafter propose a quality-adjusted price-cap scheme that allows to pursue the regulatory objective. To avoid redundancy, we content ourselves with presenting the (somewhat more typical) situation in which, at the concerned target, the incumbent serves high-valuation consumers and the competitor low-valuation consumers (i.e. we take $k=I$ and $j=E$ ). It is however noteworthy that arguments carry over, mutatis mutandis, in the converse case ${ }^{13}$.

\subsection{The ideal quality-adjusted price cap}

Suppose first that the regulatory target is given by conditions (13) and (14). Assume that the incumbent is left free to choose both price and quality, provided a price-andquality cap is satisfied. Formally, let firm $I$ pin down the pair $\left(p_{I}, q_{I}\right)$ that maximizes its profit $\pi_{I}(\mathbf{p}, \mathbf{q})$ subject to the constraint

$$
\alpha p_{I}-\beta q_{I} \leq P+\gamma p_{E}-\delta q_{E}
$$

This is a modified (single-product) version of the standard quality-adjusted price cap elaborated by De Fraja and Iozzi [19] for (multi-product) monopoly regulation. The novelty is that it is extended to account for the strategic interactions that take place between competitors in a Stackelberg market. As in the standard version, as long as $\alpha>0$, the regulatory constraint is tightened by an increase in price $p_{I}$. With $\beta>0$, it is relaxed by an increase in quality $q_{I}$. In addition, the right-hand side of the cap is designed to explicitly embody the competitor's choices. With $\gamma<0$, a raise in the rival price $p_{E}$ tightens the constraint; with $\delta<0$, a raise in the rival quality $q_{E}$ relaxes it.

When the regulatory target is given by (15) and (16), the extension terms in the righthand side of (capS) are unnecessary, provided that the incumbent behaves myopically.

\footnotetext{
${ }^{13}$ This case is formally treated in Appendix A.2.2 and B.1.2.
} 
Thus, in a Nash-Cournot duopoly, $\gamma=\delta=0$ and, despite the presence of a competitor, the price cap comes back to the more standard quality-adjusted formulation

$$
\alpha p_{I}-\beta q_{I} \leq P
$$

Let $\mu$ the Lagrange multiplier associated with either (capS) or (capN). The following proposition summarizes how the regulator should set the weights and the ceiling $P$ for the target to be enforced ${ }^{14}$.

Proposition 2 Define:

$$
\nu \equiv\left(\frac{\partial X_{E}^{R} / \partial p_{I}}{-\partial X_{E}^{R} / \partial p_{E}}\right)=\left[1+\frac{q_{I}^{R}-q_{E}^{R}}{x_{m}^{R} f\left(\theta_{m}^{R}\right)} \int_{\underline{\theta}}^{\theta_{m}^{R}}\left(\frac{-\partial x_{E}^{R}}{\partial p_{E}}\right) f(\theta) d \theta\right]^{-1}
$$

When the regulatory target is given by (13) and (14), in the ideal (capS) weights are set as:

$$
\begin{aligned}
\alpha^{S} & =X_{I}^{R}-\nu X_{E}^{R} \\
\beta^{S} & =\widetilde{\theta}_{I}^{R} X_{I}^{R}-\theta_{m}^{R} \nu X_{E}^{R} \\
\gamma^{S} & =-X_{E}^{R} \\
\delta^{S} & =-\widetilde{\theta}_{E}^{R} X_{E}^{R} .
\end{aligned}
$$

When the regulatory target is given by (15) and (16), in the ideal (capN) weights are set as:

$$
\begin{aligned}
\alpha^{N} & =X_{I}^{R}-\nu X_{E}^{R} \\
\beta^{N} & =\widetilde{\theta}_{I}^{R} X_{I}^{R}-\theta_{m}^{R} \nu X_{E}^{R} .
\end{aligned}
$$

$P^{z}$ is chosen so that $\pi_{I}\left(\mathbf{p}^{R}, \mathbf{q}^{R}\right)=0, \forall z \in\{S, N\}$.

Proposition 2 tells that, for (capS) (resp. (capN)) to implement the optimal bundle characterized by (13) and (14) (resp. (15) and (16)), it suffices $(i)$ to set coefficients as in (aS) to (dS) (resp. (aN) and (bN)) and (ii) to decrease $P$ enough to wash out the profit of firm $I$. The presence of the superscript $R$ indicates that the exact values are those obtained at the optimal price and quality, which are decentralized under the (partial) regulatory regime $^{15}$.

The first, perhaps most striking, point to be made is that the optimal weights to be attached to the incumbent's price and quality are to be determined in the same way in the Stackelberg and in the Nash-Cournot case ((aS) is analogous to (aN) and (bS) to (bN)).

\footnotetext{
${ }^{14}$ See Appendix A.1.2 and A.2.2 for mathematical details.

${ }^{15}$ To save over notation, here and elsewhere in the text, we append the superscript $R$ to denote the optimal values, whatever the target they refer to. Remark, however, that this is not meant to suggest that optimal values are the same in the different cases.
} 
According to (aS) and (aN), the appropriate weight for the incumbent's price is given by the difference between two terms. The first term is the regulated firm's quantity evaluated at $\left(\mathbf{p}^{R}, \mathbf{q}^{R}\right)$, namely $X_{I}^{R}$. The second term consists in firm $E^{\prime}$ s quantity evaluated at $\left(\mathbf{p}^{R}, \mathbf{q}^{R}\right)$, namely $X_{E}^{R}$, as multiplied by the coefficient $\nu$ that reflects product differentiation. Observe that, in general, $\nu$ is strictly smaller than one ${ }^{16}$. Thus, firm $I^{\prime}$ s output is given a larger relevance than firm $E^{\prime}$ s output in the composition of the price weight. That is to say, the price weight $\alpha$ is obtained by subtracting from the regulated firm's quantity $X_{I}^{R}$ (the "standard" weight in cap formulae) a fraction of the quantity of the (unregulated) competitor, $\nu X_{E}^{R}$.

Similarly, the quality weight $\beta$, as defined by (bS) and (bN), is given by the difference between two terms. The first term, $\widetilde{\theta}_{I}^{R} X_{I}^{R}$, is an aggregate measure of the quality appreciation by firm $I^{\prime}$ s consumers. The second term is linked to the appreciation of quality by firm $E^{\prime}$ s consumers. As the sole marginal clients of firm $E$ are concerned by changes in $q_{I}$, the quality appreciation refers to $\theta_{m}^{R}$ and not to $\widetilde{\theta}_{E}^{R}$. Note that, if prices and qualities are observable (as assumed), unlike $\widetilde{\theta}_{E}^{R}$, the parameter $\theta_{m}^{R}$ can be easily computed. Interestingly enough, this marginal quality valuation is to be multiplied by $\nu X_{E}^{R}$, the exact same part of $\alpha$ that refers to firm $E$. The coefficient is to be calculated by using the whole demand for firm $E^{\prime}$ s product, $X_{E}^{R}$ (which is possibly observable), and not the consumption by firm $E^{\prime}$ s marginal clients (which is not).

The downsizing of $X_{E}^{R}$ and $\theta_{m}^{R} X_{E}^{R}$ in the expressions of $\alpha$ and $\beta$ respectively, which depends upon the coefficient $\nu$, relates to three elements. Firstly, ceteris paribus, the smaller the quality spread between products $\left(q_{I}^{R}-q_{E}^{R}\right)$, the larger $\nu$ (and so the smaller $\alpha$ and $\beta$ ). This suggests that less regulatory pressure needs to be exerted on firm $I$ when products are not very differentiated. Indeed, in that case, the leader is disciplined by fierce competitive pressure. Secondly, the higher the marginal demand $x_{m}^{R} f\left(\theta_{m}^{R}\right)$, the weaker the regulation. A similar argument applies here: having a large amount of individuals indifferent between operators signals that, given prices and qualities, products are almost "perfect substitutes", so that competition is again a substitute for regulation. Lastly, the smaller the term $\int_{\underline{\theta}}^{\theta_{m}^{R}}\left(\frac{-\partial x_{E}^{R}}{\partial p_{E}}\right) f(\theta) d \theta$, the higher $\nu$, meaning that a relatively soft regulation is required when the entrant has market power.

All in all, a clear message can be drawn by looking at the optimal values of $\alpha$ and $\beta$ characterized in Proposition 2. First of all, neither in the Stackelberg nor in the NashCournot case the regulator can neglect the presence of the competitor to properly regulate the incumbent. Second, the larger the market share of the unregulated firm, the lower the pressure that is necessary to impose on the regulated firm. In fact, if $X^{R}$ denotes the market size at $\left(\mathbf{p}^{R}, \mathbf{q}^{R}\right)$, the optimal weight attached to price $p_{I}$ in the price-cap formula can be rewritten $\alpha=\left[X^{R}-(1+\nu) X_{E}^{R}\right]$. As $\nu>0$, this also means that competition

\footnotetext{
${ }^{16}$ As from the definition in Proposition 2 and from Appendix A.1.2, the coefficient $\nu$ is the ratio between the marginal variation in $X_{F}^{R}$ induced by an increase in $p_{L}$ and the (absolute value of the) overall (marginal and inframarginal) variation in the same quantity $X_{F}^{R}$ as induced by an increase in $p_{F}$. In general, the ratio is strictly smaller than 1 because the (cross) effect of price $p_{L}$ on the follower's demand is lower than the (own) effect of price $p_{F}$. Its specific magnitude depends on the difference between cross and own-price effects. The sole case in which $\nu$ equals 1 is the unit demand case, as mentioned later in the text.
} 
has a bigger impact on markets than it appears when considering the sole market share of the unregulated firm $\left(X_{E}^{R}\right)$. This further evidences an important feature of our cap: it accounts for (and adapts to) the transition from regulated monopolies to increasingly more competitive markets.

From Proposition 2 it also emerges that, when the regulator points to the Stackelberg benchmark, the magnitude of the competitive effect and the relevance of the competitor's product quality for consumers should be considered not only to fix the weights of the regulated price and quality, but also to tighten/relax the overall ceiling. More precisely, according to $(\mathrm{gS})$, the larger the entrant's demand $\left(X_{E}^{R}\right)$ the more a raise in $p_{E}$ tightens the cap. On the other hand, (dS) reveals that the larger the aggregate quality appreciation by firm $E^{\prime}$ s consumers $\left(\widetilde{\theta}_{E}^{R} X_{E}^{R}\right)$ the more a raise in $q_{E}$ relaxes it.

The predictions of our analysis confirm Brennan [12]'s intuition that, in the presence of competitors endowed with market power, a cap on the incumbent's price cannot be optimally calibrated on the sole incumbent's output because, unlike in the presence of a passive fringe, the marginal welfare effect of a price variation is affected by the marginal change in the competitors' output. Actually, Proposition 2 formalizes Brennan [12]'s conclusion for price-cap regulation of the incumbent in Stackelberg and Nash-Cournot oligopolies. Besides, it extends that conclusion to environments where not only price but also quality is regulated.

A peculiar case arises when customers allocate a single consumption unit to their preferred operator ${ }^{17}$. The peculiarity is that, being $\nu$ exactly equal to 1 , the Nash-Cournot target can be pursued by simply adopting a "standard" quality-adjusted price-cap. The latter is a cap of the form $\left(p_{I}-\widetilde{\theta}_{I}^{R} q_{I}\right) \leq \bar{p}$, in which the incumbent's price is replaced by its consumers' generalized price. This recipe is equally suitable for decentralization of the Stackelberg target, provided the incumbent's decisions have no impact on the entrant's quality choice ${ }^{18}$. The striking aspect is that the optimal policy does account for the impact of regulation on the whole industry and, yet, the regulatory target can be enforced by looking at the incumbent only. Despite this result holds for very specific environments, it may prove important for regulatory practice if, in such environments, relevant markets are difficult to define.

\subsection{Implementation and robustness issues}

At the implementation stage, the first concern of the regulator is to identify the target that is possible to pursue, which in turn dictates which policy is to be adopted. Actually, this depends upon the incumbent's strategic behaviour. The target is represented by (13)

\footnotetext{
${ }^{17}$ Examples of unit demand can be found in commuter transport. A commuter allocates his/her consumption unit (i.e. the trip to be made daily to reach the workplace) to the transport mode that makes him/her best off among all available alternatives. Note also that a quality attribute like travel time is both observable and verifiable, hence it can be used for regulatory purposes.

${ }^{18}$ This could be the case in environments where unregulated operators offer some minimum quality level that does not react to variations in the incumbent's price and quality, say, because they obey some given standard or for technological reasons. See Appendix A.1.4 for mathematical details on the unit demand case in the Stackelberg framework.
} 
and (14) when firm $I$ acts as a Stackelberg leader, in which case (capS) is to be adopted. The target is given by (15) and (16) when firm I plays à la Nash-Cournot, in which case $(\mathrm{capN})$ is the appropriate policy.

From a social perspective, the Nash-Cournot target is less desirable than the Stackelberg target in that it embodies less information about firms' reactions. Yet, it is the target that is feasible whenever the regulated firm is not in a position to move first and/or to commit in the market game. This evidences that the incumbent's strategic position affects the regulator's capability to pursue more or less ambitious objectives. Observe, however, that regulation itself may have an impact on the incumbent's strategic position. On one hand, imposing repeated regulatory revisions on the incumbent may progressively remove its strategic advantage. Conversely, by reducing the operational flexibility of the incumbent, regulation may preserve and perpetuate its commitment ability.

Actually, the intertemporal impact of regulation is not a minor aspect because, in the same vein as in Vogelsang and Finsinger [33], De Fraja and Iozzi [19], Billette de Villemeur [8] and Billette de Villemeur and Vinella $[10]^{19}$, one can conceive that the targeted pair of vectors $\left(\mathbf{p}^{R}, \mathbf{q}^{R}\right)$ be approached through an iterative process. More precisely, as for a "standard" price cap, information on past market performance can be used to update the weights in the constraint at each step; and the firm's profit can be progressively reduced by adjusting $P$ until $\pi_{I}=0$. It is worth to mention that, both in the Stackelberg and in the Nash-Cournot case, our regulatory scheme exhibits the following robustness property ${ }^{20}$.

Proposition 3 Let $W^{S}$ (resp. $W^{N}$ ) the welfare level that is achieved under (capS) (resp. $(\operatorname{capN}))$ when $P$ is set such that $\pi_{I}(\mathbf{p}, \mathbf{q})=0$. Around the optimal values characterized in Proposition 2, variations in $\alpha$ and $\beta$ have zero first-order effects:

$$
\frac{d W^{z}}{d \alpha}=0 \text { and } \frac{d W^{z}}{d \beta}=0, \quad \forall z \in\{S, N\} .
$$

According to Proposition 3, variations in the price weight and in the quality weight around their optimal values have no first-order effect on welfare. This means that the scheme is robust to the possibility that the regulator be unable to set $\alpha$ and $\beta$ exactly as dictated in Proposition 2. In other words, proper functioning of the scheme would not be undermined, should small biases appear in the weight determination. This result should reassure practitioners who might object that it would be problematic to properly set parameters in the cap, especially as far as the quality valuation (namely $\widetilde{\theta}_{I}^{R}$ and $\theta_{m}^{R}$ ) and the degree of competition are concerned.

Remark however that this last preoccupation is especially weak in the unit demand cases in which the regulator needs to determine the sole quality weight $\widetilde{\theta}_{I}^{R}$. Actually,

\footnotetext{
${ }^{19}$ The works of Vogelsang and Finsinger [33], De Fraja and Iozzi [19] and Billette de Villemeur [8] belong to the wide family of papers that elaborate converging schemes of price regulation under monopoly. Billette de Villemeur and Vinella [10] propose a converging scheme of partial quantity regulation under Cournot (quantity) competition.

${ }^{20}$ See Appendix A.1.3 and A.2.3 for mathematical details.
} 
in these cases, the incentive scheme reduces to a single parameter, to be exogenously set. If quality is to be considered by the regulator, then this parameter is the simplest information one can think of. Indeed, it is an average marginal valuation of quality by the incumbent's consumers, upon which the regulator may legitimately and more easily collect data $^{21}$. Thus, along the current practice, we suggest that the regulator estimate the social valuation of quality and use this estimate as an attribute in the implementation scheme ${ }^{22}$. With a quality value that is fixed and publicly available, the scheme would be transparent and little prone to manipulations, hence more likely to attract public consensus.

\section{Concluding remarks}

There are essentially two insights to be drawn from our analysis.

First, in a partially regulated oligopoly, the regulatory agency should be able to hinge upon information on the whole industry. Information about the sole regulated firm does not appear to allow for efficient regulation, in general. In the price-and-quality cap we have looked at, appropriate weights depend on the (optimal) quantities provided by both the regulated incumbent and the entrant, despite the latter is not directly concerned by regulation. These results hold true whether firms compete à la Stackelberg or à la NashCournot.

Second, under both kinds of competition, price-and-quality cap regulation is robust to small errors in the determination of the weights to be attached to the regulated firm's decision variables. It thus appears reasonable to hinge upon such regulatory mechanisms to account for the quality dimensions of the products sold in vertically differentiated markets, despite they may rely upon (possibly imperfect) statistical estimates.

We have considered settings in which quality is as flexible as price. However, for some quality dimensions, adjustments can take longer than for price. It is reasonable to think that, in liberalized markets in which this occurs, incumbents still enjoy a strategic advantage in quality setting vis-à-vis new competitors. By contrast, this advantage is less likely to survive in price setting. Such situations can be represented by a three-stage game in which a sequential quality choice by the incumbent and then by the entrant is followed by (simultaneous) price competition ${ }^{23}$. In this game, unlike in the Stackelberg context, the entrant anticipates the impact its quality decision will have on both its own and the incumbent's price ${ }^{24}$. For this reason, the regulatory target differs from those we

\footnotetext{
${ }^{21}$ For instance, stated preferences can be (and are indeed largely) used to form time value estimates in passenger transport sectors.

${ }^{22}$ This is in contrast with the implementation scheme proposed by De Fraja and Iozzi [19] for monopoly regulation, in which quality valuation is endogenously determined by computing at each step consumer marginal surplus $(\partial V / \partial q)$. In the multi-product environment the authors explore, this creates a problem in terms of convergence of the regulatory algorithm to the second-best monopoly prices and qualities. This problem is circumvented by introducing an additional constraint that further limits the regulated firm's choices.

${ }^{23}$ We are thankful to the associate editor for suggesting us to consider this setup.

${ }^{24}$ When quality is a longer-run decision variable, as compared to price, it represents the very strategic instrument for firms. This is the situation Grilo [21] and Cremer, De Rycke and Grimaud [15] represent in their mixed oligopoly models. However, these models differ from the three-stage game we refer to in
} 
have presented ${ }^{25}$. One might thus expect a different regulatory policy to be required for its decentralization. Perhaps somewhat surprisingly, it turns out that both the structure of the regulatory constraint and the optimal composition of the weights exactly replicate those of the Stackelberg context. Moreover, the scheme maintains the same robustness property at the implementation stage. This points to the conclusion that the regulatory recipe we provide, though far from general, applies to a larger variety of situations than our approach might seem to suggest at first.

Nonetheless, there still is a long way to go for a full understanding of price-andquality regulation of vertically differentiated oligopolies. Our study represents an initial undertaking in that direction. Because our goal was to pinpoint how competition with a strategic entrant affects the incumbent's regulation, we have found it useful to look at single-product firms as a first step. However, in regulated utilities, operators often provide several goods/services. To account for this circumstance, the analysis should be extended to the case of multi-product firms, which has been addressed only for monopolies so far. Moreover, we have taken firms to behave non cooperatively. In practice, they might have an incentive to collude so as to undo the regulatory policy. That asymmetrically regulated firms can profitably coordinate against the regulator is shown by Aubert and Pouyet [4] in Bayesian environments with adverse selection. It would thus be interesting to study the ideal price-and-quality cap with regard to collusive settings. This is left for further research.

\footnotetext{
that the public firm is taken to have no strategic advantage over the private firm. Competitors play a two-stage game, in which they set qualities anticipating the impact their choices will have on prices. In Grilo [21], it emerges that first best is viable in mixed duopolies, whereas it is not in private regulated duopolies, because public managers are better informed than regulators. First best entails also in Cremer, De Rycke and Grimaud [15] as long as the budget constraint of the public firm does not bind. Otherwise, a second-best outcome arises, which is still preferable to that a private duopoly would yield. By contrast, in the partial regulatory setting we have considered, the first-best outcome is beyond reach even without budget requirements and under "perfect" regulation.

${ }^{25}$ More precisely, albeit the competitors' pricing rules are the same as in the Cournot framework, the rules that characterize the competitors' qualities differs from both the Cournot and the Stackelberg counterparts. See Appendix B for mathematical details about the three-stage game described in the text.
} 


\section{References}

[1] Armstrong, M., S. Cowan and J. Vickers (1994), Regulatory Reform. Economic Analysis and British Experience, The MIT Press, Cambridge

[2] Armstrong, M., and D. E. M. Sappington, "Recent Developments in the Theory of Regulation", in Armstrong, M., and R. Porter (ed.), Handbook of Industrial Organization, Vol.III, Elsevier, North-Holland

[3] Armstrong, M., and D. E. M. Sappington (2006), "Regulation, Competition and Liberalization", Journal of Economic Literature, 44(2), 325-366

[4] Aubert, C., and J. Pouyet (2006), "Incomplete Regulation, Market Competition and Collusion", Review of Economic Design, 10(2), 113-142

[5] Beato, P., and A. Mas-Colell (1984), "The Marginal Cost Pricing Rule as a Regulation Mechanism in Mixed Markets", in Marchand, M., P. Pestieau and H. Tulkens (eds.), The Performance of Public Enterprises, North-Holland, Amsterdam, pp.81-101

[6] Bergantino, A. S., E. Billette de Villemeur and A. Vinella (2006), "A Model of Partial Regulation in the Maritime Ferry Industry", Working Paper SERIES No.10

[7] Biglaiser, G., and C. A. Ma (1995), "Regulating a dominant firm: unknown demand and industry structure", RAND Journal of Economics, 26 (1): 1-19

[8] Billette de Villemeur, E. (2004), "Regulation in the Air: Price-and-Frequency Caps", Transportation Research Part E, 40: 465-476

[9] Billette de Villemeur, E., H. Cremer, B. Roy and J. Toledano (2003), "Optimal Pricing and Price-Cap Regulation in the Postal Sector", Journal of Regulatory Economics, 24 (1): 49-62

[10] Billette de Villemeur, E., and A. Vinella (2007), "Regolamentazione parziale di quantità", Rivista di Politica Economica, Vol. VII-VIII, 79-113

[11] Bös, D. (1989), Public Enterprise Economics, North-Holland, Amsterdam, 2nd revised edition

[12] Brennan, T. (1989), "Regulating by Capping Prices", Journal of Regulatory Economics, 1(2), 133-147

[13] CARAT (2005), Quantitative impact of the regulation on TV advertising markets in EU Member States, EEA countries, the new Member States and a number of third party countries, Italy - Report 3 (9/18), Stages B and C, V.3.3

[14] Crampes, C., and M. Moreaux (2001), "Water Resource and Power Generation", International Journal of Industrial Organization, 19, 975-997

[15] Cremer, H., M. De Rycke and A. Grimaud (1997), "Service Quality, Competition, and Regulatory Policies in the Postal Sector", Kluwer Academic Publishers

[16] Cremer, H., J.-P. Florens, A. Grimaud, S. Marcy, B. Roy and J. Toledano (2001), "Entry and Competition in the Postal Market: Foundations for the Construction of Entry Scenarios", Journal of Regulatory Economics, 19(2), 107-121

[17] Crew, M. A., and D. Parker (2006), International Handbook on Economic Regulation, Edward Elgar Publishing, Cheltenham, UK, \& Northampton, US

[18] De Fraja, G., and E. Iossa (1998), "Price Caps and Output Floors: A Comparison of Simple Regulatory Rules", The Economic Journal, 108: 1404-1421 
[19] De Fraja, G., and A. Iozzi (2008), "The Quest for Quality: A Dynamic Regulatory Mechanism for Optimum Quality", Journal of Economics 85 Management Strategy, 17(4), 1011-1040

[20] De Fraja, G., and A. Iozzi (2001), "Short-Term and Long-Term Effects of Price Cap Regulation", International Journal of Developing Planning Literature, 16, 51-59 (special issue on "The Telecommunications in the 21st Century", edited by W. J. Baumol and V. A. Beker)

[21] Grilo I. (1994), "Mixed Duopoly under Vertical Differentiation", Annales d'Economie et de Statistique, 33, 91-112

[22] Helm, D., and T. Jenkinson (1998), Ed., Competition in Regulated Industries, Oxford University Press, Oxford

[23] Iozzi, A., J. Poritz and E. Valentini (2002), "Social Preferences and Price Cap Regulation", Journal of Public Economic Theory, 4 (1), 93-112

[24] Laffont, J.-J., and J. Tirole (1996), "Creating Competition through Interconnection: Theory and Practice", Journal of Regulatory Economics, 10: 227-256

[25] Laffont, J.-J., and J. Tirole (1993), A Theory of Incentives in Procurement and Regulation, The MIT Press, Cambridge

[26] Legambiente (2008), Pendolaria. La libertà di muoversi in treno

[27] Martimort, D., and W. Sand-Zantman (2006), "Signaling and the Design of Delegated Management Contracts for Public Utilities", The RAND Journal of Economics, 37(4), 763-782

[28] Nett, L. (1993), "Mixed Oligopoly with Homogeneous Goods", Annales de l'Economie Publique, 367-393

[29] Rovizzi, L. and D. Thompson (1992), "The Regulation of Product Quality in the Public Utilities and the Citizen's Charter", Fiscal Studies 13 (3), 74-95

[30] Sappington, D. (2005), "Regulating Service Quality: A Survey", Journal of Regulatory Economics, 27(2), 123-154

[31] Sappington, D. (2003), "The Effects of Incentive Regulation on Retail Telephone Service Quality in the United States", Review of Network Economics, 2(4), 355-37

[32] Vogelsang, I. (2002), "Incentive Regulation and Competition in Public Utility Markets: A 20-Year Perspective", Journal of Regulatory Economics, 22(1), 5-27

[33] Vogelsang, I., and G. Finsinger (1979), "Regulatory Adjustment Process for Optimal Pricing by Multiproduct Firms", Bell Journal of Economics, 10, 157-171

[34] Weisman, D. (2005), "Price Regulation and Quality", Information Economics and Policy, 17(2), 165-174 


\section{A The Stackelberg and the Nash-Cournot framework}

As a first step, we propose the formal analysis for the Stackelberg framework. After characterizing the price-and-quality bundle of firm $I$ (the regulatory target), we derive the optimal weights in the price-and-quality cap, describing both the case in which $p_{I}>p_{E}$ and $q_{I}>q_{E}$ and that in which $p_{I}<p_{E}$ and $q_{I}<q_{E}$. This allows us to clarify that analogous conclusions are reached, mutatis mutandis, in either case. Thus, to avoid redundancy, we thereafter focus on the former case only. We further show that the scheme is robust to imperfections in the determination of the price and quality weight.

As a second step, we repropose the analysis for the Nash-Cournot framework, taking $p_{I}>p_{E}$ and $q_{I}>q_{E}$ for the reason previously illustrated.

\section{A.1 The Stackelberg framework}

\section{A.1.1 The regulatory benchmark}

Firm $I$ maximizes (11) subject to (12). The first-order condition with respect to $p_{I}$ is given by

$$
\frac{d \pi_{I}}{d p_{I}}=\left(\frac{-1}{1+\lambda}\right)\left(\frac{d V}{d p_{I}}+\frac{d \pi_{E}}{d p_{I}}\right) .
$$

The impact on consumer surplus of a change in $p_{I}$ can be decomposed as

$$
\frac{d V}{d p_{I}}=\frac{\partial V}{\partial p_{I}}+\frac{\partial V}{\partial p_{E}} \frac{d p_{E}}{d p_{I}}+\frac{\partial V}{\partial q_{E}} \frac{d q_{E}}{d p_{I}} .
$$

With firm $I$ serving the market segment $\left[\theta_{m}, \bar{\theta}\right]$ and firm $E$ the segment $\left[\underline{\theta}, \theta_{m}\right)$, Roy's identity yields

$$
\begin{aligned}
\frac{\partial V}{\partial p_{I}} & =-\int_{\theta_{m}}^{\bar{\theta}} x_{I}\left(p_{I}, q_{I} ; \theta\right) f(\theta) d \theta=-X_{I} \\
\frac{\partial V}{\partial p_{E}} & =-\int_{\underline{\theta}}^{\theta_{m}} x_{E}\left(p_{E}, q_{E} ; \theta\right) f(\theta) d \theta=-X_{E} \\
\frac{\partial V}{\partial q_{I}} & =\int_{\theta_{m}}^{\bar{\theta}} x_{I}\left(p_{I}, q_{I} ; \theta\right) \theta f(\theta) d \theta=\widetilde{\theta}_{I} X_{I} \\
\frac{\partial V}{\partial q_{E}} & =\int_{\underline{\theta}}^{\theta_{m}} x_{E}\left(p_{E}, q_{E} ; \theta\right) \theta f(\theta) d \theta=\widetilde{\theta}_{E} X_{E} .
\end{aligned}
$$

In the opposite case, denoting the marginal type $\theta_{m, 2}$ to avoid confusion, we would get

$$
\begin{aligned}
\frac{\partial V}{\partial p_{I}} & =-\int_{\underline{\theta}}^{\theta_{m, 2}} x_{I}\left(p_{I}, q_{I} ; \theta\right) f(\theta) d \theta=-X_{I} \\
\frac{\partial V}{\partial p_{E}} & =-\int_{\theta_{m, 2}}^{\bar{\theta}} x_{E}\left(p_{E}, q_{E} ; \theta\right) f(\theta) d \theta=-X_{E} \\
\frac{\partial V}{\partial q_{I}} & =\int_{\underline{\theta}}^{\theta_{m, 2}} x_{I}\left(p_{I}, q_{I} ; \theta\right) \theta f(\theta) d \theta=\widetilde{\theta}_{I} X_{I} \\
\frac{\partial V}{\partial q_{E}} & =\int_{\theta_{m, 2}}^{\bar{\theta}} x_{E}\left(p_{E}, q_{E} ; \theta\right) \theta f(\theta) d \theta=\widetilde{\theta}_{E} X_{E} .
\end{aligned}
$$


On the other hand, because $\left(\partial \pi_{E} / \partial p_{E}\right)=0$ and $\left(\partial \pi_{E} / \partial q_{E}\right)=0$, we can write

$$
\frac{d \pi_{E}}{d p_{I}}=\frac{\partial \pi_{E}}{\partial p_{I}}=X_{E}\left(\frac{\partial X_{E} / \partial p_{I}}{-\partial X_{E} / \partial p_{E}}\right)
$$

Plugging (19a), (19b) and (19d) into (18) and then (20) and (18) into (17), we obtain (13). by

The first-order condition with respect to $q_{I}$ for a constrained maximum of (11) is given

$$
\frac{d \pi_{I}}{d q_{I}}=\left(\frac{-1}{1+\lambda}\right)\left(\frac{d V}{d q_{I}}+\frac{\partial \pi_{E}}{\partial q_{I}}\right)
$$

A similar analysis yields the following decomposition of the variation in consumer surplus

$$
\frac{d V}{d q_{I}}=\widetilde{\theta}_{I} X_{I}-X_{E} \frac{d p_{E}}{d q_{I}}+\widetilde{\theta}_{E} X_{E} \frac{d q_{E}}{d q_{I}} .
$$

We can also write

$$
\frac{\partial \pi_{E}}{\partial q_{I}}=X_{E}\left(\frac{\partial X_{E} / \partial q_{I}}{-\partial X_{E} / \partial p_{E}}\right)
$$

Replacing (22) and (23) into (21), we ultimately obtain (14).

\section{A.1.2 The ideal price-and-quality cap}

From (13) and (14), at optimum, $\alpha$ and $\beta$ are found to be:

$$
\begin{aligned}
\alpha^{S} & =X_{I}^{R}-X_{E}^{R}\left(\frac{\partial X_{E}^{R} / \partial p_{I}}{-\partial X_{E}^{R} / \partial p_{E}}\right), \\
\beta^{S} & =\widetilde{\theta}_{I}^{R} X_{I}^{R}+X_{E}^{R}\left(\frac{\partial X_{E}^{R} / \partial q_{I}}{-\partial X_{E}^{R} / \partial p_{E}}\right) .
\end{aligned}
$$

The derivatives $\left(\partial X_{E}^{R} / \partial p_{I}\right)$ and $\left(\partial X_{E}^{R} / \partial q_{I}\right)$ reflect only marginal variations, as firm $E^{\prime} \mathrm{s}$ inframarginal customers are not concerned by changes in $p_{I}$ and $q_{I}$. The other weights in Proposition 2 follow straightforwardly.

The case of $p_{I}>p_{E}$ and $q_{I}>q_{E}$ The marginal type is given by $\theta_{m} \equiv\left(\frac{p_{I}-p_{E}}{q_{I}-q_{E}}\right)$. Hence, at optimum, we can write

$$
\frac{\partial X_{E}^{R}}{\partial p_{I}}=x_{m}^{R} f\left(\theta_{m}^{R}\right) \frac{\partial \theta_{m}^{R}}{\partial p_{I}}=\frac{x_{m}^{R} f\left(\theta_{m}^{R}\right)}{q_{I}^{R}-q_{E}^{R}}
$$

together with

$$
\begin{aligned}
\frac{\partial X_{E}^{R}}{\partial q_{I}} & =x_{m}^{R} f\left(\theta_{m}^{R}\right) \frac{\partial \theta_{m}^{R}}{\partial q_{I}} \\
& =-\theta_{m}^{R} \frac{x_{m}^{R} f\left(\theta_{m}^{R}\right)}{q_{I}^{R}-q_{E}^{R}}=-\theta_{m}^{R} \frac{\partial X_{E}^{R}}{\partial p_{I}}
\end{aligned}
$$


where $x_{m}^{R} f\left(\theta_{m}^{R}\right)$ measures consumption by marginal clients. We also have

$$
\begin{aligned}
\frac{\partial X_{E}^{R}}{\partial p_{E}} & =\int_{\underline{\theta}}^{\theta_{m}^{R}} \frac{\partial x_{E}^{R}}{\partial p_{E}} f(\theta) d \theta+x_{m}^{R} f\left(\theta_{m}^{R}\right) \frac{\partial \theta_{m}^{R}}{\partial p_{E}} \\
& =\int_{\underline{\theta}}^{\theta_{m}^{R}} \frac{\partial x_{E}^{R}}{\partial p_{E}} f(\theta) d \theta-\frac{x_{m}^{R} f\left(\theta_{m}^{R}\right)}{q_{I}^{R}-q_{E}^{R}} .
\end{aligned}
$$

Thus

$$
\begin{aligned}
\frac{\partial X_{E}^{R} / \partial p_{I}}{-\partial X_{E}^{R} / \partial p_{E}} & =\frac{x_{m}^{R} f\left(\theta_{m}^{R}\right) /\left(q_{I}^{R}-q_{E}^{R}\right)}{\int_{\underline{\theta}}^{\theta_{m}^{R}}\left(\frac{-\partial x_{E}^{R}}{\partial p_{E}}\right) f(\theta) d \theta+\frac{x_{m}^{R} f\left(\theta_{m}^{R}\right)}{q_{I}^{R}-q_{E}^{R}}}=\nu, \\
\frac{\partial X_{E}^{R} / \partial q_{I}}{-\partial X_{E}^{R} / \partial p_{E}} & =-\theta_{m}^{R}\left(\frac{\partial X_{E}^{R} / \partial p_{I}}{-\partial X_{E}^{R} / \partial p_{E}}\right)=-\theta_{m}^{R} \nu .
\end{aligned}
$$

The optimal weights $\alpha^{S}$ and $\beta^{S}$ can thus be rewritten as

$$
\begin{aligned}
\alpha^{S} & =X_{I}^{R}-\nu X_{E}^{R}, \\
\beta^{S} & =\widetilde{\theta}_{I}^{R} X_{I}^{R}-\theta_{m}^{R} \nu X_{E}^{R} .
\end{aligned}
$$

Finally, if $P^{S}$ is chosen so that $\pi^{I}=0$, then it must be the case that

$$
\mu^{S}=\frac{1}{1+\lambda^{R}}
$$

The case of $p_{I}<p_{E}$ and $q_{I}<q_{E}$ The marginal type is given by $\theta_{m^{\prime}}=\left(\frac{p_{E}-p_{I}}{q_{E}-q_{I}}\right)$. Hence, at optimum, we can write

$$
\begin{aligned}
\frac{\partial X_{E}^{R}}{\partial p_{I}} & =\frac{\partial}{\partial p_{I}}\left(\int_{\theta_{m^{\prime}}^{R}}^{\bar{\theta}} x_{E}^{R} f(\theta) d \theta\right) \\
& =-x_{m^{\prime}}^{R} f\left(\theta_{m^{\prime}}^{R}\right) \frac{\partial \theta_{m^{\prime}}^{R}}{\partial p_{I}}=\frac{x_{m^{\prime}}^{R} f\left(\theta_{m^{\prime}}^{R}\right)}{q_{E}^{R}-q_{I}^{R}}
\end{aligned}
$$

together with

$$
\begin{aligned}
\frac{\partial X_{E}^{R}}{\partial q_{I}} & =\frac{\partial}{\partial q_{I}}\left(\int_{\theta_{m^{\prime}}^{R}}^{\bar{\theta}} x_{E}^{R} f(\theta) d \theta\right) \\
& =-x_{m^{\prime}}^{R} f\left(\theta_{m^{\prime}}^{R}\right) \frac{\partial \theta_{m^{\prime}}^{R}}{\partial q_{I}} \\
& =-\theta_{m^{\prime}}^{R} \frac{x_{m^{\prime}}^{R} f\left(\theta_{m^{\prime}}^{R}\right)}{q_{E}^{R}-q_{I}^{R}}=-\theta_{m^{\prime}}^{R} \frac{\partial X_{E}^{R}}{\partial p_{I}}
\end{aligned}
$$


We further have

$$
\begin{aligned}
\frac{\partial X_{E}^{R}}{\partial p_{E}} & =\int_{\theta_{m^{\prime}}^{R}}^{\bar{\theta}} \frac{\partial x_{E}^{R}}{\partial p_{E}} f(\theta) d \theta-x_{m^{\prime}}^{R} f\left(\theta_{m^{\prime}}^{R}\right) \frac{\partial \theta_{m^{\prime}}^{R}}{\partial p_{E}} \\
& =\int_{\theta_{m^{\prime}}^{R}}^{\bar{\theta}} \frac{\partial x_{E}^{R}}{\partial p_{E}} f(\theta) d \theta-\frac{x_{m^{\prime}}^{R} f\left(\theta_{m^{\prime}}^{R}\right)}{q_{E}^{R}-q_{I}^{R}} .
\end{aligned}
$$

Thus

$$
\begin{aligned}
\frac{\partial X_{E}^{R} / \partial p_{I}}{-\partial X_{E}^{R} / \partial p_{E}} & =\frac{x_{m^{\prime}}^{R} f\left(\theta_{m^{\prime}}^{R}\right) /\left(q_{E}^{R}-q_{I}^{R}\right)}{\int_{\underline{\theta}}^{\theta_{m^{\prime}}^{R}}\left(\frac{-\partial x_{E}^{R}}{\partial p_{E}}\right) f(\theta) d \theta+\frac{x_{m^{\prime}}^{R} f\left(\theta_{m^{\prime}}^{R}\right)}{q_{E}^{R}-q_{I}^{R}}}=\nu^{\prime}, \\
\frac{\partial X_{E}^{R} / \partial q_{I}}{-\partial X_{E}^{R} / \partial p_{E}} & =-\theta_{m^{\prime}}^{R}\left(\frac{\partial X_{E}^{R} / \partial p_{I}}{-\partial X_{E}^{R} / \partial p_{E}}\right)=-\theta_{m^{\prime}}^{R} \nu^{\prime} .
\end{aligned}
$$

The optimal weights $\alpha^{S}$ and $\beta^{S}$ can thus be rewritten as

$$
\begin{aligned}
\alpha^{S} & =X_{I}^{R}-\nu^{\prime} X_{E}^{R}, \\
\beta^{S} & =\widetilde{\theta}_{I}^{R} X_{I}^{R}-\theta_{m^{\prime}}^{R} \nu^{\prime} X_{E}^{R},
\end{aligned}
$$

which expression do not differ from the one obtained in the case $p_{I}>p_{E}$ and $q_{I}>q_{E}$.

\section{A.1.3 Robustness of the scheme}

Let us focus on the case of $p_{I}>p_{E}$ and $q_{I}>q_{E}$. As $\pi_{I}^{R}=0$, we can compute

$$
\frac{d W^{R}}{d \alpha}=\frac{d}{d \alpha}\left(V^{R}+\pi_{E}^{R}\right) .
$$

Omitting superscripts for sake of shortness, we have

$$
\begin{aligned}
\frac{d V}{d \alpha} & =\left(\frac{\partial V}{\partial p_{I}}+\frac{\partial V}{\partial p_{E}} \frac{d p_{E}}{d p_{I}}+\frac{\partial V}{\partial q_{E}} \frac{d q_{E}}{d p_{I}}\right) \frac{d p_{I}}{d \alpha}+\left(\frac{\partial V}{\partial q_{I}}+\frac{\partial V}{\partial p_{E}} \frac{d p_{E}}{d q_{I}}+\frac{\partial V}{\partial q_{E}} \frac{d q_{E}}{d q_{I}}\right) \frac{d q_{I}}{d \alpha} \\
& =\left(-X_{I}-X_{E} \frac{d p_{E}}{d p_{I}}+\widetilde{\theta}_{E} X_{E} \frac{d q_{E}}{d p_{I}}\right) \frac{d p_{I}}{d \alpha}+\left(\widetilde{\theta}_{I} X_{I}-X_{E} \frac{d p_{E}}{d q_{I}}+\widetilde{\theta}_{E} X_{E} \frac{d q_{E}}{d q_{I}}\right) \frac{d q_{I}}{d \alpha}
\end{aligned}
$$

together with

$$
\begin{aligned}
\frac{d \pi_{E}}{d \alpha} & =\frac{\partial \pi_{E}}{\partial p_{I}} \frac{d p_{I}}{d \alpha}+\frac{\partial \pi_{E}}{\partial q_{I}} \frac{d q_{I}}{d \alpha} \\
& =X_{E}\left(\frac{\partial X_{E} / \partial p_{I}}{-\partial X_{E} / \partial p_{E}}\right) \frac{d p_{I}}{d \alpha}+X_{E}\left(\frac{\partial X_{E} / \partial q_{I}}{-\partial X_{E} / \partial p_{E}}\right) \frac{d q_{I}}{d \alpha}
\end{aligned}
$$

Hence, we can write

$$
\begin{aligned}
\frac{d W}{d \alpha}= & {\left[-X_{I}-X_{E} \frac{d p_{E}}{d p_{I}}+\widetilde{\theta}_{E} X_{E} \frac{d q_{E}}{d p_{I}}+X_{E}\left(\frac{\partial X_{E} / \partial p_{I}}{-\partial X_{E} / \partial p_{E}}\right)\right] \frac{d p_{I}}{d \alpha} } \\
& +\left[\widetilde{\theta}_{I} X_{I}-X_{E} \frac{d p_{E}}{d q_{I}}+\widetilde{\theta}_{E} X_{E} \frac{d q_{E}}{d q_{I}}+X_{E}\left(\frac{\partial X_{E} / \partial q_{I}}{-\partial X_{E} / \partial p_{E}}\right)\right] \frac{d q_{I}}{d \alpha}
\end{aligned}
$$


Proceeding similarly with $\beta$, we can also write

$$
\begin{aligned}
\frac{d W}{d \beta}= & \frac{d}{d \beta}\left(V+\pi_{E}\right) \\
= & {\left[-X_{I}-X_{E} \frac{d p_{E}}{d p_{I}}+\widetilde{\theta}_{E} X_{E} \frac{d q_{E}}{d p_{I}}+X_{E}\left(\frac{\partial X_{E} / \partial p_{I}}{-\partial X_{E} / \partial p_{E}}\right)\right] \frac{d p_{I}}{d \beta} } \\
& +\left[\widetilde{\theta}_{I} X_{I}-X_{E} \frac{d p_{E}}{d q_{I}}+\widetilde{\theta}_{E} X_{E} \frac{d q_{E}}{d q_{I}}+X_{E}\left(\frac{\partial X_{E} / \partial q_{I}}{-\partial X_{E} / \partial p_{E}}\right)\right] \frac{d q_{I}}{d \beta} .
\end{aligned}
$$

Imperfections in $\alpha$ With $\pi_{I}^{R}=0$, still omitting superscripts, we can write

$$
\frac{d \pi_{I}}{d p_{I}} \frac{d p_{I}}{d \alpha}+\frac{d \pi_{I}}{d q_{I}} \frac{d q_{I}}{d \alpha}=0
$$

which yields $\frac{d p_{I}}{d \alpha}=-\left(\frac{d \pi_{I} / d q_{I}}{d \pi_{I} / d p_{I}}\right) \frac{d q_{I}}{d \alpha}$. The firm's first-order conditions with respect to $p_{I}$ and $q_{I}$ are written

$$
\begin{aligned}
& \frac{d \pi_{I}}{d p_{I}}=\mu\left(\alpha-\gamma \frac{d p_{E}}{d p_{I}}+\delta \frac{d q_{E}}{d p_{I}}\right) \\
& \frac{d \pi_{I}}{d q_{I}}=-\mu\left(\beta+\gamma \frac{d p_{E}}{d q_{I}}-\delta \frac{d q_{E}}{d q_{I}}\right) .
\end{aligned}
$$

Hence, we can write

$$
\frac{d p_{I}}{d \alpha}=\frac{\beta+\gamma \frac{d p_{E}}{d q_{I}}-\delta \frac{d q_{E}}{d q_{I}}}{\alpha-\gamma \frac{d p_{E}}{d p_{I}}+\delta \frac{d q_{E}}{d p_{I}}} \frac{d q_{I}}{d \alpha} .
$$

For the optimal values of the weights, as defined by (aS)-(dS), we have

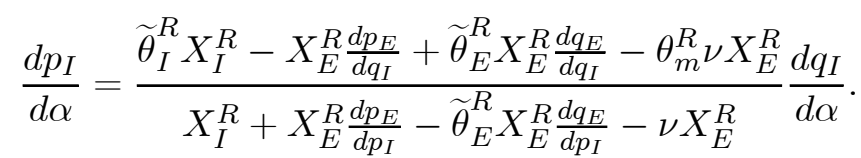

Plug this into (32) and use (27a) and (27b) to obtain $\frac{d W^{R}}{d \alpha}=0$.

Imperfections in $\beta$ With $\pi_{I}^{R}=0$, omitting superscripts again, we can write

$$
\frac{d \pi_{I}}{d p_{I}} \frac{d p_{I}}{d \beta}+\frac{d \pi_{I}}{d q_{I}} \frac{d q_{I}}{d \beta}=0
$$

which yields $\frac{d p_{I}}{d \beta}=-\left(\frac{d \pi_{I} / d q_{I}}{d \pi_{I} / d p_{I}}\right) \frac{d q_{I}}{d \beta}$. Replacing from (34) and (35) returns

$$
\frac{d p_{I}}{d \beta}=\frac{\beta+\gamma \frac{d p_{E}}{d q_{I}}-\delta \frac{d q_{E}}{d q_{I}}}{\alpha-\gamma \frac{d p_{E}}{d p_{I}}+\delta \frac{d q_{E}}{d p_{I}}} \frac{d q_{I}}{d \beta}
$$

Substituting into (33), for the optimal values of the weights, as defined by (aS)-(dS), we obtain $\frac{d W}{d \beta}^{R}=0$. 


\section{A.1.4 The case of unit demand}

With unit demand, $\nu=1$ and the price weight becomes $\alpha \equiv\left(X_{I}^{R}-X_{E}^{R}\right)$ and the quality weight $\beta \equiv\left(\widetilde{\theta}_{I}^{R} X_{I}^{R}-\theta_{m}^{R} X_{E}^{R}\right)$. Using these results, recalling that $\left(p_{I}-\theta_{m}^{R} q_{I}\right)=$ $\left(p_{E}-\theta_{m}^{R} q_{E}\right)$ and noticing that, with $p_{I}>p_{E}$ and $q_{I}>q_{E}$, we now have $X_{E}^{R}=\int_{\underline{\theta}}^{\theta_{m}^{R}} f(\theta) d \theta$ $=F\left(\theta_{m}^{R}\right)$ and $\widetilde{\theta}_{E}^{R}=\int_{\underline{\theta}}^{\theta_{m}^{R}} \frac{\theta f(\theta)}{F\left(\theta_{m}^{R}\right)} d \theta$, the regulatory constraint becomes

$$
\widetilde{p}_{I} X_{I}^{R} \leq P-\left(\theta_{m}^{R}-\widetilde{\theta}_{E}^{R}\right) q_{E} X_{E}^{R}=P-q_{E} \int_{\underline{\theta}}^{\theta_{m}^{R}} F(\theta) d \theta .
$$

When the incumbent's choices have no impact on $q_{E}$, this reduces to $\left(p_{I}-\widetilde{\theta}_{I}^{R} q_{I}\right) \leq \bar{p}$.

\section{A.2 The Nash-Cournot framework}

\section{A.2.1 The regulatory benchmark}

The first-order condition with respect to $p_{I}$ for a constrained maximum of (11) is written

$$
\frac{\partial \pi_{I}}{\partial p_{I}}=\left(\frac{1}{1+\lambda}\right)\left[X_{I}-X_{E}\left(\frac{\partial X_{E} / \partial p_{I}}{-\partial X_{E} / \partial p_{E}}\right)\right]
$$

The first-order condition with respect to $q_{I}$ for a constrained maximum of (11) is written

$$
\frac{\partial \pi_{I}}{\partial q_{I}}=\left(\frac{-1}{1+\lambda}\right)\left[\tilde{\theta}_{I} X_{I}+X_{E}\left(\frac{\partial X_{E} / \partial q_{I}}{-\partial X_{E} / \partial p_{E}}\right)\right]
$$

\section{A.2.2 The ideal price-and-quality cap}

The price-and-quality constraint reduces to

$$
\alpha p_{I}-\beta q_{I} \leq P .
$$

With $p_{I}>p_{E}$ and $q_{I}>q_{E}$, the optimal price weight is still given by

$$
\begin{aligned}
\alpha^{N} & =X_{I}^{R}-X_{E}^{R}\left(\frac{\partial X_{E}^{R} / \partial p_{I}}{-\partial X_{E}^{R} / \partial p_{E}}\right) \\
& \equiv X_{I}^{R}-\nu X_{E}^{R},
\end{aligned}
$$

where the superscript $N$ is appended to indicate the Nash game. The optimal quality weight is still given by

$$
\begin{aligned}
\beta^{N} & =\tilde{\theta}_{I}^{R} X_{I}^{R}+X_{E}^{R}\left(\frac{\partial X_{E}^{R} / \partial q_{I}}{-\partial X_{E}^{R} / \partial p_{E}}\right) \\
& \equiv \widetilde{\theta}_{I}^{R} X_{I}^{R}-\theta_{m}^{R} \nu X_{E}^{R} .
\end{aligned}
$$

Finally, if $P^{N}$ is chosen so that $\pi_{I}=0$, then it must be the case that

$$
\mu^{N}=\frac{1}{1+\lambda^{R}} .
$$




\section{A.2.3 Robustness of the scheme}

Still focusing on the case of $p_{I}>p_{E}$ and $q_{I}>q_{E}$ and omitting superscripts for sake of shortness, we now have

$$
\begin{aligned}
\frac{d V}{d \alpha} & =\frac{\partial V}{\partial p_{I}} \frac{d p_{I}}{d \alpha}+\frac{\partial V}{\partial q_{I}} \frac{d q_{I}}{d \alpha} \\
& =-X_{I} \frac{d p_{I}}{d \alpha}+\widetilde{\theta}_{I} X_{I} \frac{d q_{I}}{d \alpha}
\end{aligned}
$$

together with

$$
\begin{aligned}
\frac{d \pi_{E}}{d \alpha} & =\frac{\partial \pi_{E}}{\partial p_{I}} \frac{d p_{I}}{d \alpha}+\frac{\partial \pi_{E}}{\partial q_{I}} \frac{d q_{I}}{d \alpha} \\
& =X_{E}\left(\frac{\partial X_{E} / \partial p_{I}}{-\partial X_{E} / \partial p_{E}}\right) \frac{d p_{I}}{d \alpha}+X_{E}\left(\frac{\partial X_{E} / \partial q_{I}}{-\partial X_{E} / \partial p_{E}}\right) \frac{d q_{I}}{d \alpha}
\end{aligned}
$$

Hence, we can write

$$
\frac{d W}{d \alpha}=\left[-X_{I}+X_{E}\left(\frac{\partial X_{E} / \partial p_{I}}{-\partial X_{E} / \partial p_{E}}\right)\right] \frac{d p_{I}}{d \alpha}+\left[\widetilde{\theta}_{I} X_{I}+X_{E}\left(\frac{\partial X_{E} / \partial q_{I}}{-\partial X_{E} / \partial p_{E}}\right)\right] \frac{d q_{I}}{d \alpha} .
$$

Similarly,

$$
\frac{d W}{d \beta}=\left[-X_{I}+X_{E}\left(\frac{\partial X_{E} / \partial p_{I}}{-\partial X_{E} / \partial p_{E}}\right)\right] \frac{d p_{I}}{d \beta}+\left[\widetilde{\theta}_{I} X_{I}+X_{E}\left(\frac{\partial X_{E} / \partial q_{I}}{-\partial X_{E} / \partial p_{E}}\right)\right] \frac{d q_{I}}{d \beta} .
$$

Imperfections in $\alpha$ The firm's first-order conditions with respect to $p_{I}$ and $q_{I}$ are written

$$
\begin{array}{ll}
\frac{d \pi_{I}}{d p_{I}}=\mu \alpha^{N} & (\mathrm{pL}-\text { cap-N) } \\
\frac{d \pi_{I}}{d q_{I}}=-\mu \beta^{N} . & (\mathrm{qL}-\text { cap-N) }
\end{array}
$$

As $\frac{d p_{I}}{d \alpha}=-\left(\frac{d \pi_{I} / d q_{I}}{d \pi_{I} / d p_{I}}\right) \frac{d q_{I}}{d \alpha}$, we can write $\frac{d p_{I}}{d \alpha}=\frac{\beta^{N}}{\alpha^{N}} \frac{d q_{I}}{d \alpha}$. For the optimal values (alpha-N) and (beta-N), we have

$$
\frac{d p_{I}}{d \alpha}=\left(\frac{\widetilde{\theta}_{I}^{R} X_{I}^{R}-\theta_{m}^{R} \nu X_{E}^{R}}{X_{I}^{R}-\nu X_{E}^{R}}\right) \frac{d q_{I}}{d \alpha} .
$$

Plugging this into the expression for $\frac{d W}{d \alpha}$ at the optimal values, we still have $\frac{d W^{R}}{d \alpha}=0$.

Imperfections in $\beta \quad$ As $\frac{d p_{I}}{d \beta}=-\left(\frac{d \pi_{I} / d q_{I}}{d \pi_{I} / d p_{I}}\right) \frac{d q_{I}}{d \beta}$, we can write $\frac{d p_{I}}{d \beta}=\frac{\beta^{N}}{\alpha^{N}} \frac{d q_{I}}{d \beta}$. Substituting into the expression for $\frac{d W}{d \beta}$, we still obtain $\frac{d W^{R}}{d \beta}=0$. 


\section{B Quality as a longer-run choice variable}

We hereafter propose the formal analysis for the situation in which quality is a longerrun choice variable, as compared to price. We first solve a three-stage game in which a sequential quality choice is followed by a simultaneous price choice, in order to characterize the regulatory target. We then proceed as in Appendix A with $p_{I}>p_{E}$ and $q_{I}>q_{E}$.

\section{B.1 The regulatory benchmark}

The game unfolds as follows. At stage 1 firm $I$ chooses $q_{I}$. At stage 2 firm $E$ chooses $q_{E}$. At stage 3 firm $I$ and firm $E$ choose $p_{I}$ and $p_{E}$ respectively. We solve the game backward taking into account that, all over the game, firm $I$ faces the constraint $\pi_{I} \geq 0$ with multiplier $\lambda$.

At stage 3, firm $E$ solves the problem

$$
\underset{p_{E}}{\operatorname{Max}} \pi_{E}\left(p_{E}, p_{I} ; q_{E}^{*}, q_{I}^{*}\right)=p_{E} X_{E}\left(p_{E}, p_{I} ; q_{E}^{*}, q_{I}^{*}\right)-C_{E}\left(X_{E}\left(p_{E}, p_{I} ; q_{E}^{*}, q_{I}^{*}\right), q_{E}^{*}\right),
$$

where a star is appended to indicate previous choices. The first-order condition with respect to $p_{E}$ is given by

$$
p_{E}-\frac{\partial C_{E}}{\partial X_{E}}=\frac{X_{E}}{-\partial X_{E} / \partial p_{E}} .
$$

Simultaneously, firm $I$ chooses $p_{I}$ facing the objective function

$$
W\left(p_{I}, p_{E} ; q_{I}^{*}, q_{E}^{*}\right)=V\left(p_{I}, p_{E} ; q_{I}^{*}, q_{E}^{*}\right)+\pi_{I}\left(p_{I}, p_{E} ; q_{I}^{*}, q_{E}^{*}\right)+\pi_{E}\left(p_{E}, p_{I} ; q_{E}^{*}, q_{I}^{*}\right) .
$$

Incorporating (36), the optimal choice is characterized by

$$
\frac{\partial \pi_{I}}{\partial p_{I}}=\left(\frac{1}{1+\lambda}\right)\left[X_{I}-X_{E}\left(\frac{\partial X_{E} / \partial p_{I}}{-\partial X_{E} / \partial p_{E}}\right)\right]
$$

At stage 2, firm $E$ solves the problem

$$
\underset{q_{E}}{M_{E x}} \pi_{E}\left(p_{E}, p_{I} ; q_{E}, q_{I}^{*}\right)=p_{E} X_{E}\left(p_{E}, p_{I} ; q_{E}, q_{I}^{*}\right)-C_{E}\left(X_{E}\left(p_{E}, p_{I} ; q_{E}, q_{I}^{*}\right), q_{E}^{*}\right) .
$$

Firm $E$ anticipates that the choice of $q_{E}$ will affect the choice of $p_{E}$ and $p_{I}$ at stage 3 . The first-order condition with respect to $q_{E}$ is given by

$$
\left(p_{E}-\frac{\partial C_{E}}{\partial X_{E}}\right)\left(\frac{\partial X_{E}}{\partial q_{E}}+\frac{\partial X_{E}}{\partial p_{E}} \frac{d p_{E}}{d q_{E}}+\frac{\partial X_{E}}{\partial p_{I}} \frac{d p_{I}}{d q_{E}}\right)+X_{E} \frac{d p_{E}}{d q_{E}}-\frac{\partial C_{E}}{\partial q_{E}}=0 .
$$

Recalling (36), replacing above and manipulating, we get

$$
\frac{X_{E}}{-\partial X_{E} / \partial p_{E}}\left(\frac{\partial X_{E}}{\partial q_{E}}+\frac{\partial X_{E}}{\partial p_{I}} \frac{d p_{I}}{d q_{E}}\right)=\frac{\partial C_{E}}{\partial q_{E}} .
$$

At stage 1, firm $I$ chooses $q_{I}$ facing the objective function

$$
W\left(p_{I}, p_{E} ; q_{I}, q_{E}\right)=V\left(p_{I}, p_{E} ; q_{I}, q_{E}\right)+\pi_{I}\left(p_{I}, p_{E} ; q_{I}, q_{E}\right)+\pi_{E}\left(p_{E}, p_{I} ; q_{E}, q_{I}\right) \text {. }
$$

Firm $I$ anticipates that the choice of $q_{I}$ will have $(a)$ a direct impact on the choice of $q_{E}$ at stage $2,(b)$ a direct impact on the choice of $p_{E}$ and $p_{I}$ at stage $3,(c)$ an indirect impact on the choice of $p_{E}$ and $p_{I}$ at stage 3 through the impact on the choice of $q_{E}$ at stage 2 . 
The choice of $q_{I}$ is characterized by

$$
\frac{d V}{d q_{I}}+(1+\lambda) \frac{d \pi_{I}}{d q_{I}}+\frac{d \pi_{E}}{d q_{I}}=0 .
$$

We have

$$
\begin{aligned}
\frac{d V}{d q_{I}} & =\frac{\partial V}{\partial q_{I}}+\left(\frac{\partial V}{\partial q_{E}}+\frac{\partial V}{\partial p_{I}} \frac{d p_{I}}{d q_{E}}+\frac{\partial V}{\partial p_{E}} \frac{d p_{E}}{d q_{E}}\right) \frac{d q_{E}}{d q_{I}}+\frac{\partial V}{\partial p_{I}} \frac{d p_{I}}{d q_{I}}+\frac{\partial V}{\partial p_{E}} \frac{d p_{E}}{d q_{I}} \\
& =\tilde{\theta}_{I} X_{I}+\left(\widetilde{\theta}_{E} X_{E}-X_{I} \frac{d p_{I}}{d q_{E}}-X_{E} \frac{d p_{E}}{d q_{E}}\right) \frac{d q_{E}}{d q_{I}}-X_{I} \frac{d p_{I}}{d q_{I}}-X_{E} \frac{d p_{E}}{d q_{I}}
\end{aligned}
$$

Moreover, we can compute

$$
\begin{aligned}
\frac{d \pi_{E}}{d q_{I}} & =\frac{\partial \pi_{E}}{\partial q_{I}}+\frac{\partial \pi_{E}}{\partial q_{E}} \frac{d q_{E}}{d q_{I}}+\frac{\partial \pi_{E}}{\partial p_{E}} \frac{d p_{E}}{d q_{I}}+\frac{\partial \pi_{E}}{\partial p_{I}}\left(\frac{d p_{I}}{d q_{I}}+\frac{d p_{I}}{d q_{E}} \frac{d q_{E}}{d q_{I}}\right) \\
& =\frac{\partial \pi_{E}}{\partial q_{I}}+\frac{\partial \pi_{E}}{\partial p_{I}}\left(\frac{d p_{I}}{d q_{I}}+\frac{d p_{I}}{d q_{E}} \frac{d q_{E}}{d q_{I}}\right) \\
& =X_{E}\left[\left(\frac{\partial X_{E} / \partial q_{I}}{-\partial X_{E} / \partial p_{E}}\right)+\left(\frac{\partial X_{E} / \partial p_{I}}{-\partial X_{E} / \partial p_{E}}\right)\left(\frac{d p_{I}}{d q_{I}}+\frac{d p_{I}}{d q_{E}} \frac{d q_{E}}{d q_{I}}\right)\right]
\end{aligned}
$$

We can finally rewrite (39) as

$$
\begin{aligned}
\frac{d \pi_{I}}{d q_{I}}= & \frac{1}{1+\lambda}\left[X_{I}-X_{E}\left(\frac{\partial X_{E} / \partial p_{I}}{-\partial X_{E} / \partial p_{E}}\right)\right] \frac{d p_{I}}{d q_{I}} \\
& -\frac{1}{1+\lambda}\left[\widetilde{\theta}_{I} X_{I}+X_{E}\left(\frac{\partial X_{E} / \partial q_{I}}{-\partial X_{E} / \partial p_{E}}\right)\right. \\
& +X_{E}\left(\frac{\partial X_{E} / \partial p_{I}}{-\partial X_{E} / \partial p_{E}}\right) \frac{d p_{I}}{d q_{E}} \frac{d q_{E}}{d q_{I}}-X_{E} \frac{d p_{E}}{d q_{I}} \\
& \left.+\left(\widetilde{\theta}_{E} X_{E}-X_{I} \frac{d p_{I}}{d q_{E}}-X_{E} \frac{d p_{E}}{d q_{E}}\right) \frac{d q_{E}}{d q_{I}}\right] .
\end{aligned}
$$

Overall, the regulatory target is given by the pair of conditions (37) and (41).

\section{B.2 The ideal price-and-quality cap}

Let the constraint

$$
\alpha p_{I}-\beta q_{I} \leq P+\gamma p_{E}-\delta q_{E},
$$

Firm $I$ maximizes $\pi_{I}$ subject to (cap-III). The Lagrangian is written

$$
\ell=\pi_{I}+\mu\left(P+\gamma p_{E}-\delta q_{E}-\alpha p_{I}+\beta q_{I}\right),
$$

with $\mu$ the multiplier associated with (cap-III). We thus have

$$
\begin{aligned}
\frac{\partial \pi_{I}}{\partial p_{I}}= & \mu \alpha \\
\frac{d \pi_{I}}{d q_{I}}= & -\mu\left[\gamma\left(\frac{d p_{E}}{d q_{I}}+\frac{d p_{E}}{d q_{E}} \frac{d q_{E}}{d q_{I}}\right)-\delta \frac{d q_{E}}{d q_{I}}\right. \\
& \left.-\alpha\left(\frac{d p_{I}}{d q_{I}}+\frac{d p_{I}}{d q_{E}} \frac{d q_{E}}{d q_{I}}\right)+\beta\right] .
\end{aligned}
$$


With $p_{I}>p_{E}$ and $q_{I}>q_{E}$, the regulator should set

$$
\begin{aligned}
\alpha^{I I I} & =X_{I}^{R}-\nu X_{E}^{R} \\
\beta^{I I I} & =\widetilde{\theta}_{I}^{R} X_{I}^{R}-\theta_{m}^{R} \nu X_{E}^{R} \\
\gamma^{I I I} & =-X_{E}^{R} \\
\delta^{I I I} & =-\widetilde{\theta}_{E}^{R} X_{E}^{R}
\end{aligned}
$$

and $P^{I I I}$ such that

$$
\mu^{I I I}=\frac{1}{1+\lambda^{R}},
$$

where the superscript $I I I$ is appended to indicate the optimal values of the weights and the ceiling in the three-stage game.

\section{B.3 Robustness of the scheme}

Omitting superscripts for sake of shortness, we now have

$$
\begin{aligned}
\frac{d V}{d \alpha} & =\frac{\partial V}{\partial p_{I}} \frac{d p_{I}}{d \alpha}+\left[\frac{\partial V}{\partial q_{I}}+\frac{\partial V}{\partial q_{E}} \frac{d q_{E}}{d q_{I}}+\frac{\partial V}{\partial p_{E}}\left(\frac{d p_{E}}{d q_{I}}+\frac{d p_{E}}{d q_{E}} \frac{d q_{E}}{d q_{I}}\right)\right] \frac{d q_{I}}{d \alpha} \\
& =-X_{I} \frac{d p_{I}}{d \alpha}+\left[\widetilde{\theta}_{I} X_{I}+\widetilde{\theta}_{E} X_{E} \frac{d q_{E}}{d q_{I}}-X_{E}\left(\frac{d p_{E}}{d q_{I}}+\frac{d p_{E}}{d q_{E}} \frac{d q_{E}}{d q_{I}}\right)\right] \frac{d q_{I}}{d \alpha} .
\end{aligned}
$$

together with

$$
\begin{aligned}
\frac{d \pi_{E}}{d \alpha}= & \frac{\partial \pi_{E}}{\partial p_{I}}\left[\frac{d p_{I}}{d \alpha}+\left(\frac{d p_{I}}{d q_{I}}+\frac{d p_{I}}{d q_{E}} \frac{d q_{E}}{d q_{I}}\right) \frac{d q_{I}}{d \alpha}\right]+\frac{\partial \pi_{E}}{\partial q_{I}} \frac{d q_{I}}{d \alpha} \\
= & X_{E}\left(\frac{\partial X_{E} / \partial p_{I}}{-\partial X_{E} / \partial p_{E}}\right)\left[\frac{d p_{I}}{d \alpha}+\left(\frac{d p_{I}}{d q_{I}}+\frac{d p_{I}}{d q_{E}} \frac{d q_{E}}{d q_{I}}\right) \frac{d q_{I}}{d \alpha}\right] \\
& +X_{E}\left(\frac{\partial X_{E} / \partial q_{I}}{-\partial X_{E} / \partial p_{E}}\right) \frac{d q_{I}}{d \alpha} \\
= & X_{E}\left[\left(\frac{\partial X_{E} / \partial p_{I}}{-\partial X_{E} / \partial p_{E}}\right)\left(\frac{d p_{I}}{d q_{I}}+\frac{d p_{I}}{d q_{E}} \frac{d q_{E}}{d q_{I}}\right)\right. \\
& \left.+\left(\frac{\partial X_{E} / \partial q_{I}}{-\partial X_{E} / \partial p_{E}}\right)\right] \frac{d q_{I}}{d \alpha}+X_{E}\left(\frac{\partial X_{E} / \partial p_{I}}{-\partial X_{E} / \partial p_{E}}\right) \frac{d p_{I}}{d \alpha}
\end{aligned}
$$

Hence, we can write

$$
\begin{aligned}
\frac{d W}{d \alpha}= & {\left[X_{E}\left(\frac{\partial X_{E} / \partial p_{I}}{-\partial X_{E} / \partial p_{E}}\right)-X_{I}\right] \frac{d p_{I}}{d \alpha}+\left\{\widetilde{\theta}_{I} X_{I}+\widetilde{\theta}_{E} X_{E} \frac{d q_{E}}{d q_{I}}-X_{E}\left(\frac{d p_{E}}{d q_{I}}+\frac{d p_{E}}{d q_{E}} \frac{d q_{E}}{d q_{I}}\right)\right.} \\
& \left.+X_{E}\left[\left(\frac{\partial X_{E} / \partial p_{I}}{-\partial X_{E} / \partial p_{E}}\right)\left(\frac{d p_{I}}{d q_{I}}+\frac{d p_{I}}{d q_{E}} \frac{d q_{E}}{d q_{I}}\right)+\left(\frac{\partial X_{E} / \partial q_{I}}{-\partial X_{E} / \partial p_{E}}\right)\right]\right\} \frac{d q_{I}}{d \alpha} .
\end{aligned}
$$

Similarly,

$$
\begin{aligned}
\frac{d W}{d \beta}= & {\left[X_{E}\left(\frac{\partial X_{E} / \partial p_{I}}{-\partial X_{E} / \partial p_{E}}\right)-X_{I}\right] \frac{d p_{I}}{d \beta}+\left\{\tilde{\theta}_{I} X_{I}+\tilde{\theta}_{E} X_{E} \frac{d q_{E}}{d q_{I}}-X_{E}\left(\frac{d p_{E}}{d q_{I}}+\frac{d p_{E}}{d q_{E}} \frac{d q_{E}}{d q_{I}}\right)\right.} \\
& \left.+X_{E}\left[\left(\frac{\partial X_{E} / \partial p_{I}}{-\partial X_{E} / \partial p_{E}}\right)\left(\frac{d p_{I}}{d q_{I}}+\frac{d p_{I}}{d q_{E}} \frac{d q_{E}}{d q_{I}}\right)+\left(\frac{\partial X_{E} / \partial q_{I}}{-\partial X_{E} / \partial p_{E}}\right)\right]\right\} \frac{d q_{I}}{d \beta} .
\end{aligned}
$$




\section{B.3.1 Imperfections in $\alpha$}

From (pL-cap-III) and (qL-cap-III), we can compute

$$
\begin{aligned}
\frac{d p_{I}}{d \alpha}= & \frac{1}{\alpha}\left[\gamma\left(\frac{d p_{E}}{d q_{I}}+\frac{d p_{E}}{d q_{E}} \frac{d q_{E}}{d q_{I}}\right)-\delta \frac{d q_{E}}{d q_{I}}\right. \\
& \left.-\alpha\left(\frac{d p_{I}}{d q_{I}}+\frac{d p_{I}}{d q_{E}} \frac{d q_{E}}{d q_{I}}\right)+\beta\right] \frac{d q_{I}}{d \alpha}
\end{aligned}
$$

At the optimal values of the weights (alpha-III) - (delta-III), this becomes

$$
\begin{aligned}
\frac{d p_{I}}{d \alpha}= & \frac{1}{X_{I}^{R}-\nu X_{E}^{R}}\left[-X_{E}^{R}\left(\frac{d p_{E}}{d q_{I}}+\frac{d p_{E}}{d q_{E}} \frac{d q_{E}}{d q_{I}}\right)+\widetilde{\theta}_{E}^{R} X_{E}^{R} \frac{d q_{E}}{d q_{I}}\right. \\
& \left.-\left(X_{I}^{R}-\nu X_{E}^{R}\right)\left(\frac{d p_{I}}{d q_{I}}+\frac{d p_{I}}{d q_{E}} \frac{d q_{E}}{d q_{I}}\right)+\widetilde{\theta}_{I}^{R} X_{I}^{R}-\theta_{m}^{R} \nu X_{E}^{R}\right] \frac{d q_{I}}{d \alpha}
\end{aligned}
$$

Replacing this, we have $\frac{d W^{R}}{d \alpha}=0$.

\section{B.3.2 Imperfections in $\beta$}

From (pL-cap-III) and (qL-cap-III), we can compute

$$
\begin{aligned}
\frac{d p_{I}}{d \beta}= & \frac{1}{\alpha}\left[\gamma\left(\frac{d p_{E}}{d q_{I}}+\frac{d p_{E}}{d q_{E}} \frac{d q_{E}}{d q_{I}}\right)-\delta \frac{d q_{E}}{d q_{I}}\right. \\
& \left.-\alpha\left(\frac{d p_{I}}{d q_{I}}+\frac{d p_{I}}{d q_{E}} \frac{d q_{E}}{d q_{I}}\right)+\beta\right] \frac{d q_{I}}{d \beta}
\end{aligned}
$$

At the optimal values of the weights (alpha-III) - (delta-III), this becomes

$$
\begin{aligned}
\frac{d p_{I}}{d \beta}= & \frac{1}{X_{I}^{R}-\nu X_{E}^{R}}\left[-X_{E}^{R}\left(\frac{d p_{E}}{d q_{I}}+\frac{d p_{E}}{d q_{E}} \frac{d q_{E}}{d q_{I}}\right)+\widetilde{\theta}_{E}^{R} X_{E}^{R} \frac{d q_{E}}{d q_{I}}\right. \\
& \left.-\left(X_{I}^{R}-\nu X_{E}^{R}\right)\left(\frac{d p_{I}}{d q_{I}}+\frac{d p_{I}}{d q_{E}} \frac{d q_{E}}{d q_{I}}\right)+\widetilde{\theta}_{I}^{R} X_{I}^{R}-\theta_{m}^{R} \nu X_{E}^{R}\right] \frac{d q_{I}}{d \beta}
\end{aligned}
$$

Replacing this, we have $\frac{d W^{R}}{d \beta}=0$. 